\title{
The Optimum Output Quantity for Different Competitive Behaviors under a Fuzzy Decision Environment
}

\author{
Hong-Xia Sun, ${ }^{1}$ Yu Li, ${ }^{1}$ and Feng Wang ${ }^{2}$ \\ ${ }^{1}$ Business School, Beijing Technology and Business University, Beijing 100048, China \\ ${ }^{2}$ Shanghai Lixin University of Commerce, Shanghai 201620, China \\ Correspondence should be addressed to Hong-Xia Sun; sunhongxia@btbu.edu.cn
}

Received 13 August 2015; Revised 27 September 2015; Accepted 29 September 2015

Academic Editor: Vladimir Turetsky

Copyright (C) 2015 Hong-Xia Sun et al. This is an open access article distributed under the Creative Commons Attribution License, which permits unrestricted use, distribution, and reproduction in any medium, provided the original work is properly cited.

Due to the uncertainty of information and complexity of decision-making environment, the optimum output quantity is studied under a fuzzy decision environment. Firstly, the triangular intuitionistic fuzzy model is proposed. Secondly, the optimum output quantity is discussed for four patterns to market structure. Thirdly, the effect of fuzzy parameter on optimum output quantity and total market demand is discussed. Finally, a numerical example is given to illustrate the concrete application of the proposed model.

\section{Introduction}

In the fierce market competition, in order to gain competitive advantage and maximize profit, the optimum output quantity decision among different enterprises or coalitions in supply chain has been an important issue. In a duopoly market, two models can be used to optimize the output quantity. One is the Cournot model [1]; the other is the Stackelberg model [2]. The Cournot model forms a situation in which each firm chooses its output independently. The classical Stackelberg model is composed of one leader and one follower. The leader makes its decision taking into account the reaction of the follower. The follower, on the other hand, makes its decision assuming the leader will keep its supply quantity fixed.

The Cournot and Stackelberg models have been widely studied in the literature. Much of the literature about the Cournot model has focused on the extension, equilibrium, and application. For example, Dang et al. (2014) [3] developed the Cournot production game with multiple firms in an ambiguous decision environment, where the form of ambiguity is described by a set of fuzzy parameters. Van den Berg et al. (2012) [4] studied a dynamic Cournot duopoly in which suppliers have a limited amount of products available for two consecutive periods. Barr and Saraceno (2005) [5] examined the effects of both environmental and organizational factors on the outcome of repeated Cournot games. Guo (2010)
[6] initially proposed a one-shot decision approach to solve for the Cournot equilibrium. Guo et al. (2010) [7] further extended the method proposed in Guo [6] to a duopoly market with asymmetric possibilistic information describing the demand uncertainty only known by one firm. Colombo and Labrecciosa (2013) [8] pointed out that when the asset stock grows sufficiently fast, the dynamic Cournot game corresponds to the static Cournot solution. Fang and Shou (2015) [9] applied the Cournot model in supply chain competition. $\mathrm{Hu}$ et al. (2014) [10] developed the entry and competition of a plant factory supply chain in vegetable markets, using a Nash-Cournot model to simulate this competition. Some researchers focused on the Stackelberg model. For instance, DeMiguel and $\mathrm{Xu}$ (2009) [11] studied an oligopoly consisting of $M$ leaders and $N$ followers that supply a homogeneous product noncooperatively. De Wolf and Smeers (1997) [12] made an interesting extension of the singleleader Stackelberg-Nash-Cournot model by incorporating demand uncertainty. Nakamura (2015) [13] analyzed oneleader and multiple-follower Stackelberg games with demand uncertainty. In the research of application, Kim (2012) [14] proposed a new Cognitive Radio network spectrum sharing scheme based on the multileader multifollower Stackelberg game model. Alvarez-Vázquez et al. (2015) [15] applied the Stackelberg techniques to the environmental problem related to determining the optimal location and purification profile 
when building a new treatment plant in a wastewater depuration system. Yang and Zhou (2006) [16] analyzed the effects of the duopolistic retailers in different competitive behaviors: Cournot, Collusion, and Stackelberg on the optimal decisions of the manufacturer and the duopolistic retailers themselves.

In practical application problems, the economic assessment data, such as the fixed costs and the per unit cost, are not exact. It is necessary to consider information of market uncertainty. Liang et al. (2008) [17] developed optimum output quantity decision analysis of a duopoly market under a fuzzy environment, where the form of ambiguity is described by trapezoidal fuzzy numbers. Triangular intuitionistic fuzzy numbers (TIFNs) may express an ill-known quantity with different degree of membership and degree of nonmembership. It is an extension of triangular fuzzy number which can represent the fuzzy essence of uncertain information and depict fuzzy preference information of decision makers. Therefore, TIFNs will be used in this paper to describe the inverse demand functions of market and cost functions, and the optimum output quantity of duopoly market will be discussed.

This paper is organized as follows. In Section 2, the operations and ranking method of TIFNs are reviewed. Section 3 describes the triangular intuitionistic fuzzy model of duopoly; then the optimum output quantity is discussed for four patterns to market structure. Section 4 discusses the effect of fuzzy parameter changing on optimum output quantity and total market demand. Section 5 presents a numerical example to illustrate the concrete application of the proposed model. Conclusions are made in Section 6.

\section{Triangular Intuitionistic Fuzzy Numbers (TIFNs)}

2.1. The Definition and Operations of TIFNs. In this section, TIFNs and their operations are defined as follows.

Definition 1 (see [18]). A TIFN $\tilde{a}=\left\langle(\underline{a}, a, \bar{a}) ; \omega_{\tilde{a}}, u_{\tilde{a}}\right\rangle$ is a special intuitionistic fuzzy set on a real number set $\mathbb{R}$, whose membership function and nonmembership function are defined as follows:

$$
\begin{aligned}
& \mu_{\tilde{a}}(x)= \begin{cases}\frac{x-\underline{a}}{a-\underline{a}} w_{\tilde{a}} & \text { if } \underline{a} \leq x<a \\
w_{\tilde{a}} & \text { if } x=a \\
\frac{\bar{a}-x}{\bar{a}-a} w_{\tilde{a}} & \text { if } a<x \leq \underline{a} \\
0 & \text { if } x<\underline{a} \text { or } x>\bar{a},\end{cases} \\
& v_{\tilde{a}}(x)= \begin{cases}\frac{a-x+u_{\tilde{a}}(x-\underline{a})}{a-\underline{a}} & \text { if } \underline{a} \leq x<a \\
\frac{u_{\tilde{a}}-a+u_{\tilde{a}}(\bar{a}-x)}{\bar{a}-a} & \text { if } x=a \\
1 & \text { if } a<x \leq \underline{a}\end{cases}
\end{aligned}
$$

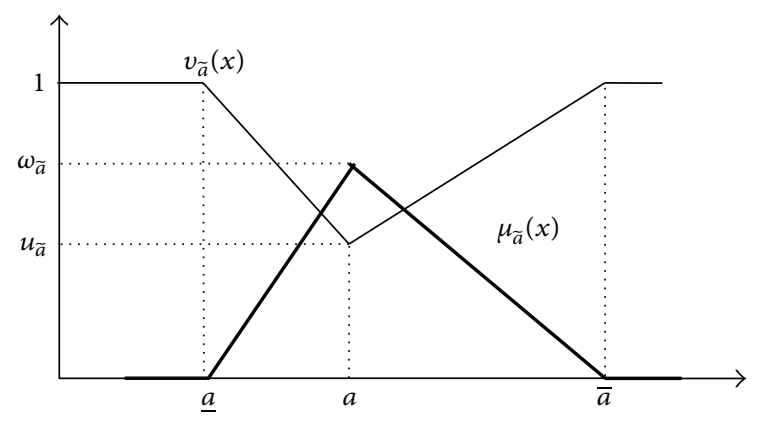

FIgURE 1: An Triangular Intuitionistic Fuzzy numbers.

respectively, depicted as in Figure 1 . The values $\omega_{\widetilde{a}}$ and $u_{\tilde{a}}$ represent the maximum degree of membership and the minimum degree of nonmembership, respectively, such that they satisfy conditions $0 \leq \omega_{\tilde{a}} \leq 1,0 \leq u_{\tilde{a}} \leq 1$, and $0 \leq \omega_{\tilde{a}}+u_{\tilde{a}} \leq 1$.

Let $\Pi_{\tilde{a}}(x)=1-\mu_{\tilde{a}}(x)-v_{\widetilde{a}}(x)$, which is called the intuitionistic fuzzy index of an element $x$ in $\tilde{a}$. It is the degree of indeterminacy membership of the element $x$ to $\widetilde{a}$.

A TIFN $\tilde{a}=\left\langle(\underline{a}, a, \bar{a}) ; \omega_{\tilde{a}}, u_{\tilde{a}}\right\rangle$ may express an ill-known quantity "approximate $a$," which is approximately equal to $a$. Namely, the ill-known quantity "approximate $a$ " is expressed using any value between $\underline{a}$ and $\bar{a}$ with different degree of membership and degree of nonmembership. The pessimistic value is $\underline{a}$ with the degree of membership 0 and the degree of nonmembership 1; the optimistic value is $\bar{a}$ with the degree of membership 0 and the degree of nonmembership 1; other value is any $x$ in the open interval $(\underline{a}, \bar{a})$ with the membership degree $\mu_{\widetilde{a}}(x)$ and the nonmembership degree $v_{\widetilde{a}}(x)$. It is easy to see that $\mu_{\tilde{a}}(x)+v_{\tilde{a}}(x)=1$ for any $x \in \mathbb{R}$ if $\omega_{\widetilde{a}}=1$ and $u_{\widetilde{a}}=$ 0 . Hence, the TIFN $\widetilde{a}=\left\langle(\underline{a}, a, \bar{a}) ; \omega_{\widetilde{a}}, u_{\widetilde{a}}\right\rangle$ degenerates to $\widetilde{a}=$ $\langle(\underline{a}, a, \bar{a}) ; 1,0\rangle$, which is just about a triangular fuzzy number. Therefore, the concept of the TIFN is a generalization of the triangular fuzzy number [19].

Definition 2 (see [20]). Let $\widetilde{a}=\left\langle(\underline{a}, a, \bar{a}) ; \omega_{\tilde{a}}, u_{\tilde{a}}\right\rangle$ and $\widetilde{b}=$ $\left\langle(\underline{b}, b, \bar{b}) ; \omega_{\tilde{b}}, u_{\tilde{b}}\right\rangle$ be two TIFNs and let $\lambda(\lambda>0)$ be a real number. The arithmetic operations over TIFNs are defined as

$$
\begin{aligned}
& \text { (1) } \tilde{a} \oplus \tilde{b}=\left\langle(\underline{a}+\underline{b}, a+b, \bar{a}+\bar{b}) ; \omega_{\tilde{a}} \wedge \omega_{\tilde{b}}, u_{\tilde{a}} \vee u_{\tilde{b}}\right\rangle, \\
& \text { (2) } \tilde{a} \ominus \tilde{b}=\left\langle(\underline{a}-\bar{b}, a-b, \bar{a}-\underline{b}) ; \omega_{\tilde{a}} \wedge \omega_{\tilde{b}}, u_{\tilde{a}} \vee u_{\tilde{b}}\right\rangle, \\
& \text { (3) } \lambda \tilde{a}=\left\langle(\lambda \underline{a}, \lambda a, \lambda \bar{a}) ; \omega_{\tilde{a}}, u_{\tilde{a}}\right\rangle,
\end{aligned}
$$

where the symbols " $\wedge$ " and " $\vee$ " are the min and $\max$ operators, respectively.

\subsection{The Ranking Methods of TIFNs}

Definition 3 (see [21]). Let $\tilde{a}=\left\langle(\underline{a}, a, \bar{a}) ; \omega_{\tilde{a}}, u_{\tilde{a}}\right\rangle$ be a TIFN. A value-index and an ambiguity-index for $\widetilde{a}$ are defined as follows:

$$
V_{\lambda}(\widetilde{a})=\frac{(\underline{a}+4 a+\bar{a})\left[\lambda \omega_{\tilde{a}}^{2}+(1-\lambda)\left(1-u_{\tilde{a}}\right)^{2}\right]}{6},
$$




$$
A_{\lambda}(\widetilde{a})=\frac{(\bar{a}-\underline{a})\left[\lambda \omega_{\tilde{a}}^{2}+(1-\lambda)\left(1-u_{\tilde{a}}\right)^{2}\right]}{6}
$$

respectively, where $\lambda \in[0,1]$ is a weight which represents the decision maker's preference information. $\lambda \in[1 / 2,1]$ shows that decision maker prefers certainty or positive feeling; $\lambda \epsilon$ $[0,1 / 2]$ shows that decision maker prefers uncertainty or negative feeling; $\lambda=1 / 2$ shows that decision maker is indifferent between certainty and uncertainty. Therefore, the value-index and the ambiguity-index may reflect the decision maker's subjectivity attitudes to the TIFN.

Let $\tilde{a}=\left\langle(\underline{a}, a, \bar{a}) ; \omega_{\tilde{a}}, u_{\tilde{a}}\right\rangle$ and $\tilde{b}=\left\langle(\underline{b}, b, \bar{b}) ; \omega_{\tilde{b}}, u_{\tilde{b}}\right\rangle$ be two TIFNs. The ranking method of TIFNs can be summarized as follows [21].

Step 1. Compare $V_{\lambda}(\widetilde{a})$ and $V_{\lambda}(\widetilde{b})$ for a given weight $\lambda$. If they are equal, then go to Step 2. Otherwise, $\operatorname{rank} \tilde{a}$ and $\tilde{b}$ according to the relative positions of $V_{\lambda}(\widetilde{a})$ and $V_{\lambda}(\widetilde{b})$. Namely, if $V_{\lambda}(\widetilde{a})>V_{\lambda}(\widetilde{b})$, then $\widetilde{a}$ is greater than $\widetilde{b}$, denoted by $\widetilde{a}>\widetilde{b}$; if $V_{\lambda}(\widetilde{a})<V_{\lambda}(\widetilde{b})$, then $\widetilde{a}$ is smaller than $\widetilde{b}$, denoted by $\widetilde{a}<\widetilde{b}$.

Step 2. Compare $A_{\lambda}(\widetilde{a})$ and $A_{\lambda}(\widetilde{b})$ for the same given $\lambda$. If they are equal, then $\tilde{a}$ and $\tilde{b}$ are equal. Otherwise, $\operatorname{rank} \tilde{a}$ and $\widetilde{b}$ according to the relative positions of $-A_{\lambda}(\widetilde{a})$ and $-A_{\lambda}(\widetilde{b})$. Namely, if $-A_{\lambda}(\widetilde{a})>-A_{\lambda}(\widetilde{b})$, then $\widetilde{a}>\widetilde{b}$; if $-A_{\lambda}(\widetilde{a})<$ $-A_{\lambda}(\tilde{b})$, then $\tilde{a}<\widetilde{b}$.

\section{Triangular Intuitionistic Fuzzy Model of Duopoly}

In a duopoly market, assume that there are two competitive players, denoted by enterprise $A$ and enterprise $B$, respectively. These two enterprises produce homogeneous products. Each enterprise's objective is to select output quantity to maximize their profit. In general, it is almost impossible to find the exact economic assessment of data for parameters' estimation in the real world. In this section, the TIFNs will be applied to study the equilibrium quantity between two enterprises. We will consider four patterns to market structure as follows:

(1) Both of enterprises $A$ and $B$ are followers.

(2) Enterprise $A$ is a leader and enterprise $B$ is a follower.

(3) Enterprise $B$ is a leader and enterprise $A$ is a follower.

(4) Both of enterprises $A$ and $B$ are leaders. follows:

Firstly, suppose the fuzzy demand price $\widetilde{p}(Q)$ is given as

$$
\widetilde{p}(Q)=\widetilde{\alpha}-\widetilde{\beta} Q=\widetilde{\alpha}-\widetilde{\beta}\left(q_{A}+q_{B}\right),
$$

where $\tilde{\alpha}=\left\langle(\underline{\alpha}, \alpha, \bar{\alpha}) ; \omega_{\tilde{\alpha}}, u_{\tilde{\alpha}}\right\rangle$ and $\widetilde{\beta}=\left\langle(\underline{\beta}, \beta, \bar{\beta}) ; \omega_{\tilde{\beta}}, u_{\tilde{\beta}}\right\rangle$ are given TIFNs. $q_{A}$ and $q_{B}$ denote the output quantities of enterprises $A$ and $B$, respectively. $Q$ is the total output quantity of duopoly market. That is, $Q=q_{A}+q_{B}$.
The fuzzy cost functions of enterprises $A$ and $B$, denoted by TIFC $_{A}$ and TIFC $_{B}$, are defined as follows:

$$
\begin{aligned}
\mathrm{TIFC}_{A} & =\widetilde{f}_{A} \oplus \widetilde{c}_{A} \otimes q_{A}, \\
\mathrm{TIFC}_{B} & =\widetilde{f}_{B} \oplus \widetilde{c}_{B} \otimes q_{B},
\end{aligned}
$$

where $\tilde{f}_{A}$ and $\tilde{f}_{B}$ denote the fuzzy fixed costs of enterprises $A$ and $B$, respectively. $\widetilde{c}_{A}$ and $\widetilde{c}_{B}$ represent the fuzzy unit variable costs, and

$$
\begin{aligned}
& \tilde{f}_{A}=\left\langle\left(\underline{f}_{A}, f_{A}, \bar{f}_{A}\right) ; \omega_{\widetilde{f}_{A}}, u_{\widetilde{f}_{A}}\right\rangle, \\
& \widetilde{c}_{A}=\left\langle\left(\underline{c}_{A}, c_{A}, \bar{c}_{A}\right) ; \omega_{\widetilde{c}_{A}}, u_{\widetilde{c}_{A}}\right\rangle, \\
& \tilde{f}_{B}=\left\langle\left(\underline{f}_{B}, f_{B}, \bar{f}_{B}\right) ; \omega_{\widetilde{f}_{B}}, u_{\tilde{f}_{B}}\right\rangle, \\
& \widetilde{c}_{B}=\left\langle\left(\underline{c}_{B}, c_{B}, \bar{c}_{B}\right) ; \omega_{\widetilde{c}_{B}}, u_{\widetilde{c}_{B}}\right\rangle .
\end{aligned}
$$

Then the fuzzy profit functions of enterprises $A$ and $B$, denoted by $\operatorname{TIF}_{A}$ and TIFח $\Pi_{B}$, can be calculated, respectively, by

$$
\begin{aligned}
& \mathrm{TIF}_{A}=\tilde{p}(Q) q_{A} \ominus \mathrm{TIFC}_{A}=\left\langle\left(\underline{\alpha} q_{A}\right.\right. \\
& -\left(q_{A}+q_{B}\right) \bar{\beta} q_{A}-\bar{f}_{A}-\bar{c}_{A} q_{A}, \alpha q_{A} \\
& -\left(q_{A}+q_{B}\right) \beta q_{A}-f_{A}-c_{A} q_{A}, \bar{\alpha} q_{A}-\left(q_{A}+q_{B}\right) \beta q_{A} \\
& \left.-\underline{f}_{A}-\underline{c}_{A} q_{A}\right) ; \min \left(\omega_{\widetilde{\alpha}}, \omega_{\widetilde{\beta}}, \omega_{\widetilde{f}_{A}}, \omega_{\widetilde{c}_{A}}\right), \\
& \left.\max \left(u_{\widetilde{\alpha}}, u_{\widetilde{\beta}}, u_{\tilde{f}_{A}}, u_{\widetilde{c}_{A}}\right)\right\rangle \text {, } \\
& \operatorname{TIF}_{B}=\tilde{p}(Q) q_{B} \ominus \operatorname{TIFC}_{B}=\left\langle\left(\underline{\alpha} q_{B}-\left(q_{A}+q_{B}\right) \bar{\beta} q_{B}\right.\right. \\
& -\bar{f}_{B}-\bar{c}_{B} q_{B}, \alpha q_{B}-\left(q_{A}+q_{B}\right) \beta q_{B}-f_{B}-c_{B} q_{B}, \bar{\alpha} q_{B} \\
& \left.-\left(q_{A}+q_{B}\right) \underline{\beta} q_{B}-\underline{f}_{B}-\underline{c}_{B} q_{B}\right) \\
& \left.\min \left(\omega_{\widetilde{\alpha}}, \omega_{\widetilde{\beta}}, \omega_{\widetilde{f}_{B}}, \omega_{\widetilde{c}_{B}}\right), \max \left(u_{\widetilde{\alpha}}, u_{\widetilde{\beta}}, u_{\widetilde{f}_{B}}, u_{\widetilde{c}_{B}}\right)\right\rangle .
\end{aligned}
$$

The fuzzy profit functions $\operatorname{TIF}_{A}$ and $\operatorname{TIF}_{B}$ are TIFNs. Their fuzziness results from the fuzzy parameters of the inverse demand function and the cost function. We utilize (2) to defuzzify the fuzzy profit function into a crisp value. If $V_{\lambda}(\widetilde{a}) \neq V_{\lambda}(\widetilde{b})$, then $V_{\lambda}\left(\operatorname{TIFM}_{A}\right)$ of $\operatorname{TIFM}_{A}$ is

$$
\begin{aligned}
V_{\lambda} & \left(\operatorname{TIF}_{A}\right)=\frac{1}{6}\left[(\underline{\alpha}+4 \alpha+\bar{\alpha}) q_{A}\right. \\
& -\left(\underline{f}_{A}+4 f_{A}+\bar{f}_{A}\right)-(\underline{\beta}+4 \beta+\bar{\beta}) q_{A}\left(q_{A}+q_{B}\right) \\
& \left.-\left(\underline{c}_{A}+4 c_{A}+\bar{c}_{A}\right) q_{A}\right]\left[\lambda \omega_{\widetilde{A}}^{2}+(1-\lambda)\left(1-u_{\widetilde{A}}\right)^{2}\right] .
\end{aligned}
$$


Similarly,

$$
\begin{aligned}
V_{\lambda} & \left(\operatorname{TIF}_{B}\right)=\frac{1}{6}\left[(\underline{\alpha}+4 \alpha+\bar{\alpha}) q_{B}\right. \\
- & \left(\underline{f}_{B}+4 f_{B}+\bar{f}_{B}\right)-(\underline{\beta}+4 \beta+\bar{\beta}) q_{B}\left(q_{A}+q_{B}\right) \\
- & \left.\left(\underline{c}_{B}+4 c_{B}+\bar{c}_{B}\right) q_{B}\right]\left[\lambda \omega_{\widetilde{B}}^{2}+(1-\lambda)\left(1-u_{\widetilde{B}}\right)^{2}\right],
\end{aligned}
$$

where

$$
\begin{aligned}
& \omega_{\widetilde{A}}=\min \left(\omega_{\widetilde{\alpha}}, \omega_{\widetilde{\beta}}, \omega_{\tilde{f}_{A}}, \omega_{\widetilde{c}_{A}}\right), \\
& u_{\widetilde{A}}=\max \left(u_{\widetilde{\alpha}}, u_{\widetilde{\beta}}, u_{\widetilde{f}_{A}}, u_{\widetilde{c}_{A}}\right), \\
& \omega_{\widetilde{B}}=\min \left(\omega_{\widetilde{\alpha}}, \omega_{\widetilde{\beta}}, \omega_{\tilde{f}_{B}}, \omega_{{\widetilde{c_{B}}}_{B}}\right), \\
& u_{\widetilde{B}}=\max \left(u_{\widetilde{\alpha}}, u_{\widetilde{\beta}}, u_{\widetilde{f}_{B}}, u_{\widetilde{c}_{B}}\right) .
\end{aligned}
$$

Remark 4. In (9), $\left[\lambda \omega_{\widetilde{A}}^{2}+(1-\lambda)\left(1-u_{\widetilde{A}}\right)^{2}\right]=0$ only if $\omega_{\widetilde{A}}=0$ and $u_{\widetilde{A}}=1$. It is a special case. In the following, we suppose that $\left[\lambda \omega_{\widetilde{A}}^{2}+(1-\lambda)\left(1-u_{\widetilde{A}}\right)^{2}\right] \neq 0$ and $\left[\lambda \omega_{\widetilde{B}}^{2}+(1-\lambda)\left(1-u_{\widetilde{B}}\right)^{2}\right] \neq 0$.

3.1. Pattern 1: Both of Enterprises $A$ and $B$ Are Followers. In this pattern, both of enterprises $A$ and $B$ independently respond with output quantities, and their optimum output quantities can be solved by the simultaneous-equation models constructed by their response functions, respectively.

By considering the maximum profit of enterprise $A$, the first-order derivative of $V_{\lambda}\left(\operatorname{TIFM}_{A}\right)$ with respect to $q_{A}$ is as follows:

$$
\begin{aligned}
& \frac{\partial V_{\lambda}\left(\operatorname{TIF}_{A}\right)}{\partial q_{A}}=\frac{1}{6}\left[(\underline{\alpha}+4 \alpha+\bar{\alpha})-2(\underline{\beta}+4 \beta+\bar{\beta}) q_{A}\right. \\
& \left.-(\underline{\beta}+4 \beta+\bar{\beta}) q_{B}-\left(\underline{c}_{A}+4 c_{A}+\bar{c}_{A}\right)\right]\left[\lambda \omega_{\widetilde{A}}^{2}\right. \\
& \left.+(1-\lambda)\left(1-u_{\widetilde{A}}\right)^{2}\right] .
\end{aligned}
$$

Solving $\partial V_{\lambda}\left(\operatorname{TIFM}_{A}\right) / \partial q_{A}=0$ will obtain the response function of enterprise $A$ :

$$
\begin{aligned}
q_{A}= & \frac{(\underline{\alpha}+4 \alpha+\bar{\alpha})-(\underline{\beta}+4 \beta+\bar{\beta}) q_{B}}{2(\underline{\beta}+4 \beta+\bar{\beta})} \\
& -\frac{\left(\underline{c}_{A}+4 c_{A}+\bar{c}_{A}\right)}{2(\underline{\beta}+4 \beta+\bar{\beta})} .
\end{aligned}
$$
$B$ :

Similarly, we can find the response function of enterprise

$$
\begin{aligned}
q_{B}= & \frac{(\underline{\alpha}+4 \alpha+\bar{\alpha})-(\underline{\beta}+4 \beta+\bar{\beta}) q_{A}}{2(\underline{\beta}+4 \beta+\bar{\beta})} \\
& -\frac{\left(\underline{c}_{B}+4 c_{B}+\bar{c}_{B}\right)}{2(\underline{\beta}+4 \beta+\bar{\beta})} .
\end{aligned}
$$

By (13) and (14), the optimum output quantities of enterprises $A$ and $B$, represented by $q_{F_{A 1}}^{*}$ and $q_{F_{B 1}}^{*}$, can be found. That is,

$$
\begin{aligned}
q_{F_{A 1}}^{*}= & \frac{(\underline{\alpha}+4 \alpha+\bar{\alpha})-2\left(\underline{c}_{A}+4 c_{A}+\bar{c}_{A}\right)}{3(\underline{\beta}+4 \beta+\bar{\beta})} \\
& +\frac{\left(\underline{c}_{B}+4 c_{B}+\bar{c}_{B}\right)}{3(\underline{\beta}+4 \beta+\bar{\beta})}, \\
q_{F_{B 1}^{*}=}^{*} & \frac{(\underline{\alpha}+4 \alpha+\bar{\alpha})-2\left(\underline{c}_{B}+4 c_{B}+\bar{c}_{B}\right)}{3(\underline{\beta}+4 \beta+\bar{\beta})} \\
& +\frac{\left(\underline{c}_{A}+4 c_{A}+\bar{c}_{A}\right)}{3(\underline{\beta}+4 \beta+\bar{\beta})} .
\end{aligned}
$$

By taking (15) into (4), the market equilibrium price $\widetilde{p}_{1}^{*}$ is solved:

$$
\begin{aligned}
\tilde{p}_{1}^{*} & =\tilde{\alpha}-\tilde{\beta}\left[\frac{2(\underline{\alpha}+4 \alpha+\bar{\alpha})-\left(\underline{c}_{B}+4 c_{B}+\bar{c}_{B}\right)}{3(\underline{\beta}+4 \beta+\bar{\beta})}\right. \\
& \left.-\frac{\left(\underline{c}_{A}+4 c_{A}+\bar{c}_{A}\right)}{3(\underline{\beta}+4 \beta+\bar{\beta})}\right] .
\end{aligned}
$$

By taking $q_{F_{A 1}}^{*}$ and $q_{F_{B 1}}^{*}$ into (7) and (8), the fuzzy maximum profits TIF $\Pi_{F_{A 1}}^{*}$ and $\operatorname{TIF}_{F_{B 1}}^{*}$ of enterprises $A$ and $B$ can be found:

$$
\begin{gathered}
\operatorname{TIF}_{F_{A 1}}^{*}=\left\langle\left(\underline{\alpha} q_{F_{A 1}}^{*}-\left(q_{F_{A 1}}^{*}+q_{F_{B 1}}^{*}\right) \bar{\beta} q_{F_{A 1}}^{*}-\bar{f}_{A}\right.\right. \\
-\bar{c}_{A} q_{F_{A 1}}^{*}, \alpha q_{F_{A 1}}^{*}-f_{A}-\left(q_{F_{A 1}}^{*}+q_{F_{B 1}}^{*}\right) \beta q_{F_{A 1}}^{*} \\
-c_{A} q_{F_{A 1}}^{*}, \bar{\alpha} q_{F_{A 1}}^{*}-\left(q_{F_{A 1}}^{*}+q_{F_{B 1}}^{*}\right) \beta q_{F_{A 1}}^{*}-\underline{f}_{A} \\
\left.\left.-\underline{c}_{A} q_{F_{A 1}}^{*}\right) ; \omega_{\widetilde{A}}, u_{\widetilde{A}}\right\rangle, \\
\operatorname{TIF} \Pi_{F_{B 1}}^{*}=\left\langle\left(\underline{\alpha}_{F_{B 1}}^{*}-\left(q_{F_{A 1}}^{*}+q_{F_{B 1}}^{*}\right) \bar{\beta} q_{F_{B 1}}^{*}-\bar{f}_{B}\right.\right. \\
-\bar{c}_{B} q_{F_{B 1}}^{*}, \alpha q_{F_{B 1}}^{*}-f_{B}-\left(q_{F_{A 1}}^{*}+q_{F_{B 1}}^{*}\right) \beta q_{F_{B 1}}^{*} \\
-c_{B} q_{F_{B 1}}^{*}, \bar{\alpha} q_{F_{B 1}^{*}}^{*}-\left(q_{F_{A 1}}^{*}+q_{F_{B 1}}^{*}\right) \underline{\beta}_{F_{B 1}}^{*}-\underline{f}_{B} \\
\left.\left.-\underline{c}_{B} q_{F_{B 1}}^{*}\right) ; \omega_{\widetilde{B}}, u_{\widetilde{B}}\right\rangle .
\end{gathered}
$$

By (17) and (18), the fuzzy total profit TIFח $\Pi_{1}^{*}$ in pattern 1 is as below:

$$
\operatorname{TIF}_{1}^{*}=\operatorname{TIF}_{F_{A 1}}^{*} \oplus \operatorname{TIF}_{F_{B 1}}^{*} .
$$

3.2. Pattern 2: Enterprise A Is a Leader and Enterprise B Is a Follower. In this pattern, the leader makes the optimum output quantity decision by considering the response function of the follower. Then, the follower decides his optimum output quantity based on the leader's decision. 
For any given $\left(q_{1}, q_{2}\right),(7)$ and (8) represent, respectively, the profits of enterprises $A$ and $B$. Enterprise $B$ observes his reaction function $q_{2}$ and adjusts his output quantity to maximize his profit, given the output quantity decision of enterprise $A$. Enterprise $A$ maximizes his profit, given the reaction function of enterprise $B$. Substituting reaction function of enterprise $B$ into (7), the fuzzy profits function $\operatorname{TIF}_{A}$ is given as

$$
\begin{aligned}
\operatorname{TIF}_{A}=\left\langle\left(\underline{\alpha} q_{A}-\left(q_{A}+\frac{(\underline{\alpha}+4 \alpha+\bar{\alpha})}{2(\underline{\beta}+4 \beta+\bar{\beta})}\right.\right.\right. & \left.-\frac{(\underline{\beta}+4 \beta+\bar{\beta}) q_{A}+\left(\underline{c}_{B}+4 c_{B}+\bar{c}_{B}\right)}{2(\underline{\beta}+4 \beta+\bar{\beta})}\right) \bar{\beta} q_{A}-\bar{f}_{A} \\
- & \bar{c}_{A} q_{A}, \alpha q_{A}-\left(q_{A}\right. \\
+ & \frac{(\underline{\alpha}+4 \alpha+\bar{\alpha})-(\underline{\beta}+4 \beta+\bar{\beta}) q_{A}}{2(\underline{\beta}+4 \beta+\bar{\beta})} \\
- & \left.\frac{\left(\underline{c}_{B}+4 c_{B}+\bar{c}_{B}\right)}{2(\underline{\beta}+4 \beta+\bar{\beta})}\right) \beta q_{A}-f_{A}-c_{A} q_{A}, \bar{\alpha} q_{A}-\left(q_{A}\right. \\
+ & \frac{(\underline{\alpha}+4 \alpha+\bar{\alpha})}{2(\underline{\beta}+4 \beta+\bar{\beta})} \\
- & \left.\frac{(\underline{\beta}+4 \beta+\bar{\beta}) q_{A}+\left(\underline{c}_{B}+4 c_{B}+\bar{c}_{B}\right)}{2(\underline{\beta}+4 \beta+\bar{\beta})}\right) \underline{\beta} q_{A}-\underline{f}_{A} \\
- & \left.\left.\underline{c}_{A} q_{A}\right) ; \omega_{\widetilde{A}}, u_{\widetilde{A}}\right\rangle .
\end{aligned}
$$

Then the $V_{\lambda}\left(\operatorname{TIF}_{A}\right)$ of $\operatorname{TIF}_{A}$ is

$$
\begin{aligned}
& V_{\lambda}\left(\operatorname{TIF\Pi }_{A}\right)=\left[(\underline{\alpha}+4 \alpha+\bar{\alpha}) q_{A}-\left(\underline{f}_{A}+4 f_{A}+\bar{f}_{A}\right)\right. \\
& -\left(\underline{c}_{A}+4 c_{A}+\bar{c}_{A}\right) q_{A}-(\underline{\beta}+4 \beta+\bar{\beta}) \\
& \quad q_{A}\left(\frac{(\underline{\alpha}+4 \alpha+\bar{\alpha})-(\underline{\beta}+4 \beta+\bar{\beta}) q_{A}}{2(\underline{\beta}+4 \beta+\bar{\beta})}\right. \\
& \left.\left.-\frac{\left(\underline{c}_{B}+4 c_{B}+\bar{c}_{B}\right)}{2(\underline{\beta}+4 \beta+\bar{\beta})}+q_{A}\right)\right]\left[\lambda \omega_{\widetilde{A}}^{2}+(1-\lambda)(1\right. \\
& \left.\left.-u_{\widetilde{A}}\right)^{2}\right] .
\end{aligned}
$$

By solving $\partial V_{\lambda}\left(\operatorname{TIF} \Pi_{A}\right) / \partial q_{A}=0$, the optimum quantity $q_{L_{A 2}}^{*}$ of enterprise $A$ is as follows:

$$
\begin{aligned}
q_{L_{A 2}}^{*}= & \frac{(\underline{\alpha}+4 \alpha+\bar{\alpha})-2\left(\underline{c}_{A}+4 c_{A}+\bar{c}_{A}\right)}{2(\underline{\beta}+4 \beta+\bar{\beta})} \\
& +\frac{\left(\underline{c}_{B}+4 c_{B}+\bar{c}_{B}\right)}{2(\underline{\beta}+4 \beta+\bar{\beta})} .
\end{aligned}
$$

By taking (22) into (20), we can find the fuzzy maximum profit TIFח $\Pi_{A 2}^{*}$ of enterprise $A$ :

$$
\begin{aligned}
\operatorname{TIF} \Pi_{L_{A 2}}^{*}=\left\langle\left(\underline{\alpha} q_{L_{A 2}}^{*}-\left(q_{L_{A 2}}^{*}+\frac{(\underline{\alpha}+4 \alpha+\bar{\alpha})}{2(\underline{\beta}+4 \beta+\bar{\beta})}\right.\right.\right. \\
\left.-\frac{(\underline{\beta}+4 \beta+\bar{\beta}) q_{L_{A 2}}^{*}+\left(\underline{c}_{B}+4 c_{B}+\bar{c}_{B}\right)}{2(\underline{\beta}+4 \beta+\bar{\beta})}\right) \bar{\beta} q_{L_{A 2}}^{*} \\
-\bar{f}_{A}-\bar{c}_{A} q_{L_{A 2}}^{*}, \alpha q_{L_{A 2}}^{*}-\left(q_{L_{A 2}}^{*}\right. \\
+\frac{(\underline{\alpha}+4 \alpha+\bar{\alpha})-(\underline{\beta}+4 \beta+\bar{\beta}) q_{L_{A 2}}^{*}}{2(\underline{\beta}+4 \beta+\bar{\beta})} \\
\left.-\frac{\left(\underline{c}_{B}+4 c_{B}+\bar{c}_{B}\right)}{2(\underline{\beta}+4 \beta+\bar{\beta})}\right) \beta q_{L_{A 2}}^{*}-f_{A}-c_{A} q_{L_{A 2}}^{*}, \bar{\alpha} q_{L_{A 2}}^{*} \\
-\left(q_{L_{A 2}}^{*}+\frac{(\underline{\alpha}+4 \alpha+\bar{\alpha})}{2(\underline{\beta}+4 \beta+\bar{\beta})}\right. \\
\left.-\frac{(\underline{\beta}+4 \beta+\bar{\beta}) q_{L_{A 2}}^{*}+\left(\underline{c}_{B}+4 c_{B}+\bar{c}_{B}\right)}{2(\underline{\beta}+4 \beta+\bar{\beta})}\right) \underline{\beta} q_{L_{A 2}}^{*} \\
\left.\left.-\underline{f}_{A}-\underline{c}_{A} q_{L_{A 2}}^{*}\right) ; \omega_{\widetilde{A}}, u_{\bar{A}}\right\rangle . \\
\end{aligned}
$$

By taking (22) into (14), we can find the optimum output quantity $q_{F_{B 2}}^{*}$ of enterprise $B$ :

$$
\begin{aligned}
q_{F_{B 2}}^{*}= & \frac{(\underline{\alpha}+4 \alpha+\bar{\alpha})+2\left(\underline{c}_{A}+4 c_{A}+\bar{c}_{A}\right)}{4(\underline{\beta}+4 \beta+\bar{\beta})} \\
& -\frac{3\left(\underline{c}_{B}+4 c_{B}+\bar{c}_{B}\right)}{4(\underline{\beta}+4 \beta+\bar{\beta})} .
\end{aligned}
$$


By taking (22) and (24) into (4), the market equilibrium price $\widetilde{p}_{2}^{*}$ in pattern 2 can be found:

$$
\begin{aligned}
\tilde{p}_{2}^{*} & =\widetilde{\alpha}-\tilde{\beta}\left[\frac{3(\underline{\alpha}+4 \alpha+\bar{\alpha})-2\left(\underline{c}_{A}+4 c_{A}+\bar{c}_{A}\right)}{4(\underline{\beta}+4 \beta+\bar{\beta})}\right. \\
& \left.-\frac{\left(\underline{c}_{B}+4 c_{B}+\bar{c}_{B}\right)}{4(\underline{\beta}+4 \beta+\bar{\beta})}\right] .
\end{aligned}
$$

By taking $q_{L_{A 2}}^{*}$ and $q_{F_{B 2}}^{*}$ into (8), the fuzzy maximum profit TIF $_{F_{B 2}}^{*}$ of enterprise $B$ in pattern 2 is as below:

$$
\begin{aligned}
& \operatorname{TIF}_{F_{B 2}}^{*}=\left\langle\left(\underline{\alpha} q_{F_{B 2}}^{*}-\left(q_{L_{A 2}}^{*}+q_{F_{B 2}}^{*}\right) \bar{\beta} q_{F_{B 2}}^{*}-\bar{f}_{B}\right.\right. \\
& -\bar{c}_{B} q_{F_{B 2}}^{*}, \alpha q_{F_{B 2}}^{*}-f_{B}-\left(q_{L_{A 2}}^{*}+q_{F_{B 2}}^{*}\right) \beta q_{F_{B 2}}^{*} \\
& -c_{B} q_{F_{B 2}}^{*}, \bar{\alpha} q_{F_{B 2}}^{*}-\left(q_{L_{A 2}}^{*}+q_{F_{B 2}}^{*}\right) \underline{\beta}_{F_{B 2}}^{*}-\underline{f}_{B} \\
& \left.\left.\quad-\underline{c}_{B} q_{F_{B 2}}^{*}\right) ; \omega_{\widetilde{B}}, u_{\widetilde{B}}\right\rangle .
\end{aligned}
$$

Then the fuzzy total profit $\operatorname{TIF\Pi }_{2}^{*}$ in pattern 2 is

$$
\mathrm{TIF}_{2}^{*}=\operatorname{TIF}_{L_{A 2}}^{*} \oplus \operatorname{TIF}_{F_{B 2}}^{*} .
$$

3.3. Pattern 3: Enterprise B Is a Leader and Enterprise A Is a Follower. Similar to the analysis of pattern 2, the following results can be obtained.

The optimum output quantities of enterprises $A$ and $B$ are

$$
\begin{aligned}
q_{F_{A 3}}^{*}= & \frac{(\underline{\alpha}+4 \alpha+\bar{\alpha})+2\left(\underline{c}_{B}+4 c_{B}+\bar{c}_{B}\right)}{4(\underline{\beta}+4 \beta+\bar{\beta})} \\
& -\frac{3\left(\underline{c}_{A}+4 c_{A}+\bar{c}_{A}\right)}{4(\underline{\beta}+4 \beta+\bar{\beta})}, \\
q_{L_{B 3}}^{*}= & \frac{(\underline{\alpha}+4 \alpha+\bar{\alpha})-2\left(\underline{c}_{B}+4 c_{B}+\bar{c}_{B}\right)}{2(\underline{\beta}+4 \beta+\bar{\beta})} \\
& +\frac{\left(\underline{c}_{A}+4 c_{A}+\bar{c}_{A}\right)}{2(\underline{\beta}+4 \beta+\bar{\beta})} .
\end{aligned}
$$

The market equilibrium price $\widetilde{p}_{3}^{*}$ in pattern 3 is

$$
\begin{aligned}
\widetilde{p}_{3}^{*} & =\widetilde{\alpha}-\widetilde{\beta}\left[\frac{3(\underline{\alpha}+4 \alpha+\bar{\alpha})-2\left(\underline{c}_{B}+4 c_{B}+\bar{c}_{B}\right)}{4(\underline{\beta}+4 \beta+\bar{\beta})}\right. \\
& \left.-\frac{\left(\underline{c}_{A}+4 c_{A}+\bar{c}_{A}\right)}{4(\underline{\beta}+4 \beta+\bar{\beta})}\right] .
\end{aligned}
$$

The fuzzy maximum profits of enterprises $A$ and $B$ are

$$
\begin{aligned}
& \operatorname{TIF}_{F_{A 3}}^{*}=\left\langle\left(\underline{\alpha} q_{F_{A 3}}^{*}-\left(q_{L_{B 3}}^{*}+q_{F_{A 3}}^{*}\right) \bar{\beta} q_{F_{A 3}}^{*}-\bar{f}_{A}\right.\right. \\
& -\bar{c}_{A} q_{F_{A 3}}^{*}, \alpha q_{F_{A 3}}^{*}-f_{A}-\left(q_{L_{B 3}}^{*}+q_{F_{A 3}}^{*}\right) \beta q_{F_{A 3}}^{*} \\
& -c_{A} q_{F_{A 3}}^{*}, \bar{\alpha} q_{F_{A 3}}^{*}-\left(q_{L_{B 3}}^{*}+q_{F_{A 3}}^{*}\right) \beta q_{F_{A 3}}^{*}-\underline{f}_{A} \\
& \left.\left.-\underline{c}_{A} q_{F_{A 3}}^{*}\right) ; \omega_{\widetilde{A}}, u_{\widetilde{A}}\right\rangle,
\end{aligned}
$$$$
\operatorname{TIF}_{L_{B 3}}^{*}=\left\langle\left(\alpha q_{L_{B 3}}^{*}-\left(q_{L_{B 3}}^{*}+\frac{(\underline{\alpha}+4 \alpha+\bar{\alpha})}{2(\underline{\beta}+4 \beta+\bar{\beta})}\right.\right.\right.
$$$$
\left.-\frac{(\underline{\beta}+4 \beta+\bar{\beta}) q_{L_{B 3}}^{*}+\left(\underline{c}_{A}+4 c_{A}+\bar{c}_{A}\right)}{2(\underline{\beta}+4 \beta+\bar{\beta})}\right) \bar{\beta} q_{L_{B 3}^{*}}
$$$$
-\bar{f}_{B}-\bar{c}_{B} q_{L_{B 3}}^{*}, \alpha q_{L_{B 3}}^{*}-\left(q_{L_{B 3}}^{*}\right.
$$$$
+\frac{(\underline{\alpha}+4 \alpha+\bar{\alpha})-(\underline{\beta}+4 \beta+\bar{\beta}) q_{L_{B 3}}^{*}}{2(\underline{\beta}+4 \beta+\bar{\beta})}
$$

$$
\begin{aligned}
& \left.-\frac{\left(\underline{c}_{A}+4 c_{A}+\bar{c}_{A}\right)}{2(\underline{\beta}+4 \beta+\bar{\beta})}\right) \beta q_{L_{B 3}}^{*}-f_{B}-c_{B} q_{L_{B 3}}^{*}, \bar{\alpha} q_{L_{B 3}}^{*} \\
& -\left(q_{L_{B 3}}^{*}+\frac{(\underline{\alpha}+4 \alpha+\bar{\alpha})}{2(\underline{\beta}+4 \beta+\bar{\beta})}\right) \\
& \left.-\frac{(\underline{\beta}+4 \beta+\bar{\beta}) q_{L_{B 3}}^{*}+\left(\underline{c}_{A}+4 c_{A}+\bar{c}_{A}\right)}{2(\underline{\beta}+4 \beta+\bar{\beta})}\right) \beta q_{L_{B 3}}^{*} \\
& \left.\left.-\underline{f}_{B}-\underline{c}_{B} q_{L_{B 3}}^{*}\right) ; \omega_{\widetilde{B}}, u_{\widetilde{B}}\right\rangle .
\end{aligned}
$$

The fuzzy total profit TIF $\Pi_{3}^{*}$ in pattern 3 is

$$
\mathrm{TIF}_{3}^{*}=\operatorname{TIF}_{F_{A 3}}^{*} \oplus \operatorname{TIF}_{L_{B 3}}^{*} .
$$

3.4. Pattern 4: Both of Enterprises $A$ and B Are Leaders. In this pattern, the optimum output quantities of companies $A$ and $B$ can be achieved when they are recognized as leaders. Therefore, the optimum output quantities of the two enterprises are as below:

$$
\begin{aligned}
q_{L_{A 4}}^{*}= & q_{L_{A 2}}^{*} \\
= & \frac{(\underline{\alpha}+4 \alpha+\bar{\alpha})-2\left(\underline{c}_{A}+4 c_{A}+\bar{c}_{A}\right)}{2(\underline{\beta}+4 \beta+\bar{\beta})} \\
& +\frac{\left(\underline{c}_{B}+4 c_{B}+\bar{c}_{B}\right)}{2(\underline{\beta}+4 \beta+\bar{\beta})},
\end{aligned}
$$




$$
\begin{aligned}
q_{L_{B 4}}^{*}= & q_{L_{B 3}}^{*} \\
= & \frac{(\underline{\alpha}+4 \alpha+\bar{\alpha})-2\left(\underline{c}_{B}+4 c_{B}+\bar{c}_{B}\right)}{2(\underline{\beta}+4 \beta+\bar{\beta})} \\
& +\frac{\left(\underline{c}_{A}+4 c_{A}+\bar{c}_{A}\right)}{2(\underline{\beta}+4 \beta+\bar{\beta})} .
\end{aligned}
$$

By taking (32) and (36) into (4), we can find the market equilibrium price $\widetilde{p}_{4}^{*}$ in pattern 4 ; that is,

$$
\begin{aligned}
\tilde{p}_{4}^{*} & =\widetilde{\alpha}-\tilde{\beta}\left[\frac{2(\underline{\alpha}+4 \alpha+\bar{\alpha})-\left(\underline{c}_{B}+4 c_{B}+\bar{c}_{B}\right)}{2(\underline{\beta}+4 \beta+\bar{\beta})}\right. \\
& \left.-\frac{\left(\underline{c}_{A}+4 c_{A}+\bar{c}_{A}\right)}{2(\underline{\beta}+4 \beta+\bar{\beta})}\right] .
\end{aligned}
$$

By taking $q_{L_{A 4}}^{*}$ and $q_{L_{B 4}}^{*}$ into (7) and (8), the fuzzy maximum profits and the fuzzy total profit, TIFח ${ }_{L_{A 4}}^{*}, \operatorname{TIF}_{L_{B 4}}^{*}$, and $\mathrm{TIF}_{4}^{*}$, are as below:

$$
\begin{aligned}
& \operatorname{TIF}_{L_{A 4}}^{*}=\left\langle\left(\underline{\alpha} q_{L_{A 4}}^{*}-\left(q_{L_{A 4}}^{*}+q_{L_{B 4}}^{*}\right) \bar{\beta} q_{L_{A 4}}^{*}-\bar{f}_{A}\right.\right. \\
& -\bar{c}_{A} q_{L_{A 4}}^{*}, \alpha q_{L_{A 4}}^{*}-f_{A}-\left(q_{L_{A 4}}^{*}+q_{L_{B 4}}^{*}\right) \beta q_{L_{A 4}}^{*} \\
& -c_{A} q_{L_{A 4}}^{*}, \bar{\alpha} q_{L_{A 4}}^{*}-\left(q_{L_{A 4}}^{*}+q_{L_{B 4}}^{*}\right) \beta q_{L_{A 4}}^{*}-\underline{f}_{A} \\
& \left.\left.-\underline{c}_{A} q_{L_{A 4}}^{*}\right) ; \omega_{\widetilde{A}}, u_{\widetilde{A}}\right\rangle, \\
& \operatorname{TIF} \Pi_{L_{B 4}}^{*}=\left\langle\left(\underline{\alpha}_{L_{B 4}}^{*}-\left(q_{L_{A 4}}^{*}+q_{L_{B 4}}^{*}\right) \bar{\beta} q_{L_{B 4}}^{*}-\bar{f}_{B}\right.\right. \\
& -\bar{c}_{B} q_{L_{B 4}}^{*}, \alpha q_{L_{B 4}}^{*}-f_{B}-\left(q_{L_{A 4}}^{*}+q_{L_{B 4}}^{*}\right) \beta q_{L_{B 4}}^{*} \\
& -c_{B} q_{L_{B 4}}^{*}, \bar{\alpha} q_{L_{B 4}}^{*}-\left(q_{L_{A 4}}^{*}+q_{L_{B 4}}^{*}\right) \beta q_{L_{B 4}}^{*}-\underline{f}_{B} \\
& \left.\left.\quad-\underline{c}_{B} q_{L_{B 4}}^{*}\right) ; \omega_{\widetilde{B}}, u_{\widetilde{B}}\right\rangle, \\
& \operatorname{TIF} \Pi_{4}^{*}=\operatorname{TIF} \Pi_{L_{A 4}}^{*} \oplus \operatorname{TIF} \Pi_{L_{B 4}}^{*}
\end{aligned}
$$

\section{Effect of Fuzzy Parameters}

As discussed in Section 3, fuzzy parameters are defuzzified into a crisp value when calculating optimum output quantity. In this section, we use left and right spread of fuzzy parameters to discuss the effect of fuzzy parameter on optimum output quantity and market demand for the four competitive behaviors.

The TIFNs $\widetilde{\alpha}, \widetilde{\beta}, \widetilde{c}_{A}$, and $\widetilde{c}_{B}$ can be rewritten as follows:

$$
\begin{aligned}
\tilde{\alpha} & =\left\langle\left(\alpha-l_{\alpha}, \alpha, \alpha+r_{\alpha}\right) ; \omega_{\tilde{\alpha}}, u_{\tilde{\alpha}}\right\rangle, \\
\widetilde{\beta} & =\left\langle\left(\beta-l_{\beta}, \beta, \beta+r_{\beta}\right) ; \omega_{\widetilde{\beta}}, u_{\widetilde{\beta}}\right\rangle, \\
\widetilde{c}_{A} & =\left\langle\left(c_{A}-l_{c A}, c_{A}, c_{A}+r_{c A}\right) ; \omega_{\widetilde{c}_{A}}, u_{\widetilde{c}_{A}}\right\rangle, \\
\widetilde{c}_{B} & =\left\langle\left(c_{B}-l_{c B}, c_{B}, c_{B}+r_{c B}\right) ; \omega_{\widetilde{c}_{B}}, u_{\widetilde{c}_{B}}\right\rangle,
\end{aligned}
$$

where $l$ and $r$ represent left and right spread of fuzzy parameters, respectively.

Firstly, in pattern 1, by (15) and (36), the optimum output quantity of $A$ and $B$ can be rewritten as

$$
\begin{aligned}
q_{F_{A 1}}^{*}= & \frac{\left(6 \alpha-l_{\alpha}+r_{\alpha}\right)-2\left(6 c_{A}-l_{c_{A}}+r_{c_{A}}\right)}{3\left(6 \beta-l_{\beta}+r_{\beta}\right)} \\
& +\frac{\left(6 c_{B}-l_{c_{B}}+r_{c_{B}}\right)}{3\left(6 \beta-l_{\beta}+r_{\beta}\right)}, \\
q_{F_{B 1}}^{*}= & \frac{\left(6 \alpha-l_{\alpha}+r_{\alpha}\right)-2\left(6 c_{B}-l_{c_{B}}+r_{c_{B}}\right)}{3\left(6 \beta-l_{\beta}+r_{\beta}\right)} \\
& +\frac{\left(6 c_{A}-l_{c_{A}}+r_{c_{A}}\right)}{3\left(6 \beta-l_{\beta}+r_{\beta}\right)} .
\end{aligned}
$$

Then the total market demand is

$$
\begin{aligned}
Q_{1}^{*}= & \frac{2\left(6 \alpha-l_{\alpha}+r_{\alpha}\right)-\left(6 c_{B}-l_{c_{B}}+r_{c_{B}}\right)}{3\left(6 \beta-l_{\beta}+r_{\beta}\right)} \\
& -\frac{\left(6 c_{A}-l_{c_{A}}+r_{c_{A}}\right)}{3\left(6 \beta-l_{\beta}+r_{\beta}\right)} .
\end{aligned}
$$

In the following, the sensitivity analysis of the optimum output quantity and total market demand is discussed. to $l_{\alpha}$,

Taking the partial derivative of (37) and (38) with respect

$$
\begin{aligned}
\frac{\partial q_{F_{A 1}}^{*}}{\partial l_{\alpha}} & =\frac{\partial q_{F_{B 1}}^{*}}{\partial l_{\alpha}}=\frac{-1}{3\left(6 \beta-l_{\beta}+r_{\beta}\right)}<0, \\
\frac{\partial Q_{1}^{*}}{\partial l_{\alpha}} & =\frac{-2}{3\left(6 \beta-l_{\beta}+r_{\beta}\right)}<0
\end{aligned}
$$

which means that $q_{F_{A 1}}^{*}, q_{F_{B 1}}^{*}$, and $Q_{1}^{*}$ decrease in $l_{\alpha}$.

Similarly, we also take the partial derivative of (37) and (38) with respect to other fuzzy parameters, such as $r_{\alpha}, l_{\beta}$, $r_{\beta}, l_{c A}, r_{c A}, l_{c B}$, and $r_{c B}$. In addition, we can also analyze the sensitivity of the optimum output quantity and total market demand of enterprises $A$ or $B$ for the other patterns. The results are shown in Table 1 .

From the results, we can make three observations.

Observation 1. For enterprise $A$, no matter what kind of pattern, the optimum output quantity, $q_{A}^{*}$, increases in $l_{\beta}, r_{\alpha}$, $l_{c A}$, and $r_{c B}$ but decreases in $r_{\beta}, l_{\alpha}, r_{c A}$, and $l_{c B}$.

Observation 2. For enterprise $B$, no matter what kind of pattern, the optimum output quantity, $q_{B}^{*}$, increases in $l_{\beta}, r_{\alpha}$, $r_{c A}$, and $l_{c B}$ but decreases in $r_{\beta}, l_{\alpha}, l_{c A}$ and $r_{c B}$.

Observation 3. No matter what kind of pattern, the total market demand $Q^{*}$ increases in $r_{\alpha}, l_{\beta}, l_{c A}$, and $l_{c B}$ but decreases in $l_{\alpha}, r_{\beta}, r_{c A}$, and $r_{c B}$. 
TABLE 1: Partial derivatives of optimum output quantity and total market demand with respect to different fuzzy parameters in four patterns.

\begin{tabular}{|c|c|c|c|c|c|c|}
\hline Pattern 1 & \multicolumn{3}{|c|}{$l_{\beta}$} & \multicolumn{3}{|c|}{$r_{\beta}$} \\
\hline \multirow{2}{*}{$q_{F_{A}}^{*}$} & \multicolumn{3}{|c|}{$\underline{\left(6 \alpha-l_{\alpha}+r_{\alpha}\right)-2\left(6 c_{A}-l_{c_{A}}+r_{c_{A}}\right)+\left(6 c_{B}-l_{c_{B}}+r_{c_{B}}\right)}$} & \multicolumn{3}{|c|}{$-\left[\left(6 \alpha-l_{\alpha}+r_{\alpha}\right)-2\left(6 c_{A}-l_{c_{A}}+r_{c_{A}}\right)+\left(6 c_{B}-l_{c_{B}}+r_{c_{B}}\right)\right]$} \\
\hline & \multicolumn{3}{|c|}{$3\left(6 \beta-l_{\beta}+r_{\beta}\right)^{2}$} & \multicolumn{3}{|c|}{$3\left(6 \beta-l_{\beta}+r_{\beta}\right)^{2}$} \\
\hline \multirow{2}{*}{$q_{F_{B}}^{*}$} & \multicolumn{3}{|c|}{$\left(6 \alpha-l_{\alpha}+r_{\alpha}\right)-2\left(6 c_{B}-l_{c_{B}}+r_{c_{B}}\right)+\left(6 c_{A}-l_{c_{A}}+r_{c_{A}}\right)$} & \multicolumn{3}{|c|}{$-\left[\left(6 \alpha-l_{\alpha}+r_{\alpha}\right)-2\left(6 c_{B}-l_{c_{B}}+r_{c_{B}}\right)+\left(6 c_{A}-l_{c_{A}}+r_{c_{A}}\right)\right.$} \\
\hline & \multicolumn{3}{|c|}{$3\left(6 \beta-l_{\beta}+r_{\beta}\right)^{2}$} & \multicolumn{3}{|c|}{$3\left(6 \beta-l_{\beta}+r_{\beta}\right)^{2}$} \\
\hline \multirow{2}{*}{$Q_{1}^{*}$} & \multirow{2}{*}{\multicolumn{3}{|c|}{$\frac{2\left(6 \alpha-l_{\alpha}+r_{\alpha}\right)-\left(6 c_{B}-l_{c_{B}}+r_{c_{B}}\right)-\left(6 c_{A}-l_{c_{A}}+r_{c_{A}}\right)}{3\left(6 \beta-l_{\beta}+r_{\beta}\right)^{2}}$}} & \multicolumn{3}{|c|}{$-\left[2\left(6 \alpha-l_{\alpha}+r_{\alpha}\right)-\left(6 c_{B}-l_{c_{B}}+r_{c_{B}}\right)-\left(6 c_{A}-l_{c_{A}}+r_{c_{A}}\right)\right]$} \\
\hline & & & & \multicolumn{3}{|c|}{$3\left(6 \beta-l_{\beta}+r_{\beta}\right)^{2}$} \\
\hline Pattern 1 & $l_{\alpha}$ & $r_{\alpha}$ & $l_{c_{A}}$ & $r_{c_{A}}$ & $l_{c_{B}}$ & $r_{c_{B}}$ \\
\hline \multirow{2}{*}{$q_{F_{A}}^{*}$} & -1 & 1 & 2 & -2 & -1 & 1 \\
\hline & $\overline{3\left(6 \beta-l_{\beta}+r_{\beta}\right)}$ & $\overline{3\left(6 \beta-l_{\beta}+r_{\beta}\right)}$ & $\overline{3\left(6 \beta-l_{\beta}+r_{\beta}\right)}$ & $\overline{3\left(6 \beta-l_{\beta}+r_{\beta}\right)}$ & $\overline{3\left(6 \beta-l_{\beta}+r_{\beta}\right)}$ & $\overline{3\left(6 \beta-l_{\beta}+r_{\beta}\right)}$ \\
\hline \multirow{2}{*}{$q_{F_{B}}^{*}$} & -1 & 1 & -1 & 1 & 2 & -2 \\
\hline & $\overline{3\left(6 \beta-l_{\beta}+r_{\beta}\right)}$ & $\overline{3\left(6 \beta-l_{\beta}+r_{\beta}\right)}$ & $\overline{3\left(6 \beta-l_{\beta}+r_{\beta}\right)}$ & $\overline{3\left(6 \beta-l_{\beta}+r_{\beta}\right)}$ & $\overline{3\left(6 \beta-l_{\beta}+r_{\beta}\right)}$ & $\overline{3\left(6 \beta-l_{\beta}+r_{\beta}\right)}$ \\
\hline \multirow{2}{*}{$Q_{1}^{*}$} & -2 & 2 & 1 & -1 & 1 & -1 \\
\hline & $\overline{3\left(6 \beta-l_{\beta}+r_{\beta}\right)}$ & $\overline{3\left(6 \beta-l_{\beta}+r_{\beta}\right)}$ & $\overline{3\left(6 \beta-l_{\beta}+r_{\beta}\right)}$ & $\overline{3\left(6 \beta-l_{\beta}+r_{\beta}\right)}$ & $\overline{3\left(6 \beta-l_{\beta}+r_{\beta}\right)}$ & $\overline{3\left(6 \beta-l_{\beta}+r_{\beta}\right)}$ \\
\hline Pattern 2 & \multicolumn{3}{|c|}{$l_{\beta}$} & \multicolumn{3}{|c|}{$r_{\beta}$} \\
\hline \multirow{2}{*}{$q_{L_{A}}^{*}$} & \multicolumn{3}{|c|}{$\left(6 \alpha-l_{\alpha}+r_{\alpha}\right)-2\left(6 c_{A}-l_{c_{A}}+r_{c_{A}}\right)+\left(6 c_{B}-l_{c_{B}}+r_{c_{B}}\right)$} & \multicolumn{3}{|c|}{$-\left[\left(6 \alpha-l_{\alpha}+r_{\alpha}\right)-2\left(6 c_{A}-l_{c_{A}}+r_{c_{A}}\right)+\left(6 c_{B}-l_{c_{B}}+r_{c_{B}}\right)\right]$} \\
\hline & \multicolumn{3}{|c|}{$2\left(6 \beta-l_{\beta}+r_{\beta}\right)^{2}$} & & $2\left(6 \beta-l_{\beta}+r_{\beta}\right.$ & \\
\hline & $\left(6 \alpha-l_{\alpha}+r_{\alpha}\right)$ & $3\left(6 c_{B}-l_{c_{B}}+r_{c_{B}}\right)$ & $\left(6 c_{A}-l_{c_{A}}+r_{c_{A}}\right)$ & $-\left[\left(6 \alpha-l_{\alpha}+r\right.\right.$ & $-3\left(6 c_{B}-l_{c_{B}}+r\right.$ & $\left.\left(6 c_{A}-l_{c_{A}}+r_{c_{A}}\right)\right]$ \\
\hline$\Psi_{F_{B}}$ & & $4\left(6 \beta-l_{\beta}+r_{\beta}\right)$ & & & $8\left(6 \beta-l_{\beta}+\right.$ & \\
\hline$Q^{*}$ & $3\left(6 \alpha-l_{\alpha}+r_{\alpha}\right.$ & $-\left(6 c_{B}-l_{c_{B}}+r_{c_{B}}\right)$ & $\left(6 c_{A}-l_{c_{A}}+r_{c_{A}}\right)$ & $-\left[3\left(6 \alpha-l_{\alpha}+\right.\right.$ & $\alpha)-\left(6 c_{B}-l_{c_{B}}+r_{c}\right.$ & $\left.\left(6 c_{A}-l_{c_{A}}+r_{c_{A}}\right)\right]$ \\
\hline$Q_{2}$ & & $4\left(6 \beta-l_{\beta}+r_{\beta}\right)$ & & & $8\left(6 \beta-l_{\beta}+1\right.$ & \\
\hline Pattern 2 & $l_{\alpha}$ & $r_{\alpha}$ & $l_{c_{A}}$ & $r_{c_{A}}$ & $l_{c_{B}}$ & $r_{c_{B}}$ \\
\hline & -1 & 1 & 2 & -2 & -1 & 1 \\
\hline$q_{L_{A}}$ & $\overline{2\left(6 \beta-l_{\beta}+r_{\beta}\right)}$ & $\overline{2\left(6 \beta-l_{\beta}+r_{\beta}\right)}$ & $\overline{2\left(6 \beta-l_{\beta}+r_{\beta}\right)}$ & $\overline{2\left(6 \beta-l_{\beta}+r_{\beta}\right)}$ & $\overline{2\left(6 \beta-l_{\beta}+r_{\beta}\right)}$ & $\overline{2\left(6 \beta-l_{\beta}+r_{\beta}\right)}$ \\
\hline & -1 & 1 & -2 & 2 & 3 & -3 \\
\hline$q_{F_{B}}$ & $\overline{4\left(6 \beta-l_{\beta}+r_{\beta}\right)}$ & $\overline{4\left(6 \beta-l_{\beta}+r_{\beta}\right)}$ & $\overline{4\left(6 \beta-l_{\beta}+r_{\beta}\right)}$ & $\overline{4\left(6 \beta-l_{\beta}+r_{\beta}\right)}$ & $\overline{4\left(6 \beta-l_{\beta}+r_{\beta}\right)}$ & $\overline{4\left(6 \beta-l_{\beta}+r_{\beta}\right)}$ \\
\hline & -3 & 3 & 2 & -2 & 1 & -1 \\
\hline$Q_{2}$ & $\overline{4\left(6 \beta-l_{\beta}+r_{\beta}\right)}$ & $\overline{4\left(6 \beta-l_{\beta}+r_{\beta}\right)}$ & $\overline{4\left(6 \beta-l_{\beta}+r_{\beta}\right)}$ & $\overline{4\left(6 \beta-l_{\beta}+r_{\beta}\right)}$ & $\overline{4\left(6 \beta-l_{\beta}+r_{\beta}\right)}$ & $\overline{4\left(6 \beta-l_{\beta}+r_{\beta}\right)}$ \\
\hline Pattern 3 & & $l_{\beta}$ & & & $r_{\beta}$ & \\
\hline & $\left(6 \alpha-l_{\alpha}+r_{\alpha}\right)$ & $3\left(6 c_{A}-l_{c_{A}}+r_{c_{A}}\right)$ & $\left(6 c_{B}-l_{c_{B}}+r_{c_{B}}\right)$ & $-\left[\left(6 \alpha-l_{\alpha}+\right.\right.$ & $-3\left(6 c_{A}-l_{c_{A}}+r\right.$ & $\left.2\left(6 c_{B}-l_{c_{B}}+r_{c_{B}}\right)\right]$ \\
\hline$q_{F_{A}}$ & & $4\left(6 \beta-l_{\beta}+r_{\beta}\right)$ & & & $8\left(6 \beta-l_{\beta}+r\right.$ & \\
\hline & $\left(6 \alpha-l_{\alpha}+r_{\alpha}\right)$ & $-2\left(6 c_{B}-l_{c_{B}}+r_{c_{B}}\right)$ & $\left.6 c_{A}-l_{c_{A}}+r_{c_{A}}\right)$ & $-\left[\left(6 \alpha-l_{\alpha}+\right.\right.$ & $-2\left(6 c_{B}-l_{c_{B}}+\right.$ & $\left.\left(6 c_{A}-l_{c_{A}}+r_{c_{A}}\right)\right]$ \\
\hline$\Psi_{L_{B}}$ & & $2\left(6 \beta-l_{\beta}+r_{\beta}\right)$ & & & $2\left(6 \beta-l_{\beta}+r\right.$ & \\
\hline & $3\left(6 \alpha-l_{\alpha}+r_{\alpha}\right.$ & $-2\left(6 c_{B}-l_{c_{B}}+r_{c_{B}}\right.$ & $\left(6 c_{A}-l_{c_{A}}+r_{c_{A}}\right)$ & $-\left[3\left(6 \alpha-l_{\alpha}+\right.\right.$ & $\alpha)-2\left(6 c_{B}-l_{c_{B}}+\right.$ & $\left.\left(6 c_{A}-l_{c_{A}}+r_{c_{A}}\right)\right]$ \\
\hline$Q_{3}$ & & $4\left(6 \beta-l_{\beta}+r_{\beta}\right)$ & & & $8\left(6 \beta-l_{\beta}+r\right.$ & \\
\hline Pattern 3 & $l_{\alpha}$ & $r_{\alpha}$ & $l_{c_{A}}$ & $r_{c_{A}}$ & $l_{c_{B}}$ & $r_{c_{B}}$ \\
\hline & -1 & $\begin{array}{c}1 \\
\end{array}$ & $\begin{array}{c}3 \\
\end{array}$ & -3 & -2 & 2 \\
\hline$q_{F_{A}}$ & $\overline{4\left(6 \beta-l_{\beta}+r_{\beta}\right)}$ & $\overline{4\left(6 \beta-l_{\beta}+r_{\beta}\right)}$ & $\overline{4\left(6 \beta-l_{\beta}+r_{\beta}\right)}$ & $\overline{4\left(6 \beta-l_{\beta}+r_{\beta}\right)}$ & $\overline{4\left(6 \beta-l_{\beta}+r_{\beta}\right)}$ & $\overline{4\left(6 \beta-l_{\beta}+r_{\beta}\right)}$ \\
\hline & -1 & 1 & -1 & 1 & 2 & -2 \\
\hline$q_{L_{B}}$ & $\overline{2\left(6 \beta-l_{\beta}+r_{\beta}\right)}$ & $\overline{2\left(6 \beta-l_{\beta}+r_{\beta}\right)}$ & $\overline{2\left(6 \beta-l_{\beta}+r_{\beta}\right)}$ & $\overline{2\left(6 \beta-l_{\beta}+r_{\beta}\right)}$ & $\overline{2\left(6 \beta-l_{\beta}+r_{\beta}\right)}$ & $\overline{2\left(6 \beta-l_{\beta}+r_{\beta}\right)}$ \\
\hline & -3 & 3 & 1 & -1 & 2 & -2 \\
\hline$Q_{3}$ & $\overline{4\left(6 \beta-l_{\beta}+r_{\beta}\right)}$ & $\overline{4\left(6 \beta-l_{\beta}+r_{\beta}\right)}$ & $\overline{4\left(6 \beta-l_{\beta}+r_{\beta}\right)}$ & $4\left(6 \beta-l_{\beta}+r_{\beta}\right)$ & $4\left(6 \beta-l_{\beta}+r_{\beta}\right)$ & $4\left(6 \beta-l_{\beta}+r_{\beta}\right)$ \\
\hline
\end{tabular}


TABle 1: Continued.

\begin{tabular}{|c|c|c|c|c|c|c|}
\hline Pattern 4 & \multicolumn{3}{|c|}{$l_{\beta}$} & \multicolumn{3}{|c|}{$r_{\beta}$} \\
\hline \multirow{2}{*}{$q_{L_{A}}^{*}$} & \multicolumn{3}{|c|}{$\left(6 \alpha-l_{\alpha}+r_{\alpha}\right)-2\left(6 c_{A}-l_{c_{A}}+r_{c_{A}}\right)+\left(6 c_{B}-l_{c_{B}}+r_{c_{B}}\right)$} & \multicolumn{3}{|c|}{$-\left[\left(6 \alpha-l_{\alpha}+r_{\alpha}\right)-2\left(6 c_{A}-l_{c_{A}}+r_{c_{A}}\right)+\left(6 c_{B}-l_{c_{B}}+r_{c_{B}}\right)\right]$} \\
\hline & \multicolumn{3}{|c|}{$2\left(6 \beta-l_{\beta}+r_{\beta}\right)^{2}$} & \multicolumn{3}{|c|}{$2\left(6 \beta-l_{\beta}+r_{\beta}\right)^{2}$} \\
\hline \multirow{2}{*}{$q_{L_{B}}^{*}$} & \multicolumn{3}{|c|}{$\left(6 \alpha-l_{\alpha}+r_{\alpha}\right)-2\left(6 c_{B}-l_{c_{B}}+r_{c_{B}}\right)+\left(6 c_{A}-l_{c_{A}}+r_{c_{A}}\right)$} & \multicolumn{3}{|c|}{$-\left[\left(6 \alpha-l_{\alpha}+r_{\alpha}\right)-2\left(6 c_{B}-l_{c_{B}}+r_{c_{B}}\right)+\left(6 c_{A}-l_{c_{A}}+r_{c_{A}}\right)\right]$} \\
\hline & \multicolumn{3}{|c|}{$2\left(6 \beta-l_{\beta}+r_{\beta}\right)^{2}$} & \multicolumn{3}{|c|}{$2\left(6 \beta-l_{\beta}+r_{\beta}\right)^{2}$} \\
\hline \multirow{2}{*}{$Q_{4}^{*}$} & \multicolumn{3}{|c|}{$2\left(6 \alpha-l_{\alpha}+r_{\alpha}\right)-\left(6 c_{B}-l_{c_{B}}+r_{c_{B}}\right)-\left(6 c_{A}-l_{c_{A}}+r_{c_{A}}\right)$} & \multicolumn{3}{|c|}{$-\left[2\left(6 \alpha-l_{\alpha}+r_{\alpha}\right)-\left(6 c_{B}-l_{c_{B}}+r_{c_{B}}\right)-\left(6 c_{A}-l_{c_{A}}+r_{c_{A}}\right)\right]$} \\
\hline & \multicolumn{3}{|c|}{$2\left(6 \beta-l_{\beta}+r_{\beta}\right)^{2}$} & \multicolumn{3}{|c|}{$2\left(6 \beta-l_{\beta}+r_{\beta}\right)^{2}$} \\
\hline Pattern 4 & $l_{\alpha}$ & $r_{\alpha}$ & $l_{c_{A}}$ & $r_{c_{A}}$ & $l_{c_{B}}$ & $r_{c_{B}}$ \\
\hline \multirow{2}{*}{$q_{L_{A}}^{*}$} & -1 & 1 & 2 & -2 & -1 & 1 \\
\hline & $\overline{2\left(6 \beta-l_{\beta}+r_{\beta}\right)}$ & $\overline{2\left(6 \beta-l_{\beta}+r_{\beta}\right)}$ & $\overline{2\left(6 \beta-l_{\beta}+r_{\beta}\right)}$ & $\overline{2\left(6 \beta-l_{\beta}+r_{\beta}\right)}$ & $\overline{2\left(6 \beta-l_{\beta}+r_{\beta}\right)}$ & $2\left(6 \beta-l_{\beta}+r_{\beta}\right)$ \\
\hline \multirow{2}{*}{$q_{L_{B}}^{*}$} & -1 & 1 & -1 & 1 & 2 & -2 \\
\hline & $\overline{2\left(6 \beta-l_{\beta}+r_{\beta}\right)}$ & $\overline{2\left(6 \beta-l_{\beta}+r_{\beta}\right)}$ & $\overline{2\left(6 \beta-l_{\beta}+r_{\beta}\right)}$ & $\overline{2\left(6 \beta-l_{\beta}+r_{\beta}\right)}$ & $\overline{2\left(6 \beta-l_{\beta}+r_{\beta}\right)}$ & $2\left(6 \beta-l_{\beta}+r_{\beta}\right)$ \\
\hline \multirow{2}{*}{$Q_{4}^{*}$} & -2 & 2 & 1 & -1 & 1 & -1 \\
\hline & $2\left(6 \beta-l_{\beta}+r_{\beta}\right)$ & $2\left(6 \beta-l_{\beta}+r_{\beta}\right)$ & $2\left(6 \beta-l_{\beta}+r_{\beta}\right)$ & $2\left(6 \beta-l_{\beta}+r_{\beta}\right)$ & $2\left(6 \beta-l_{\beta}+r_{\beta}\right)$ & $2\left(6 \beta-l_{\beta}+r_{\beta}\right)$ \\
\hline
\end{tabular}

TABle 2: The optimum output quantities of the four patterns.

\begin{tabular}{llccc}
\hline Enterprise & Pattern 1 & Pattern 2 & Pattern 3 & Pattern 4 \\
\hline$A$ & $q_{F_{A 1}^{*}}^{*}=206.06$ & $q_{L_{A 2}}^{*}=309.09$ & $q_{F_{A 3}}^{*}=161.36$ & $q_{L_{A 4}}^{*}=309.09$ \\
$B$ & $q_{F_{B 1}}^{*}=178.79$ & $q_{F_{B 2}}^{*}=127.27$ & $q_{L_{B 3}}^{*}=268.18$ & $q_{L_{B 4}}^{*}=268.18$ \\
\hline
\end{tabular}

\section{Numerical Example}

5.1. Numerical Analysis of the Proposed Model. In a duopoly market, there are two enterprises, named $A$ and $B$, respectively. They competed with each other to gain the optimum output quantities. Suppose that the estimated fuzzy fixed costs, fuzzy unit variable cost, and the fuzzy parameters of market demand are given as follows:

$$
\begin{aligned}
\tilde{f}_{A} & =\langle(200,240,250) ; 0.6,0.3\rangle, \\
\tilde{f}_{B} & =\langle(180,230,240) ; 0.7,0.2\rangle, \\
\tilde{c}_{A} & =\langle(5,6,7) ; 0.9,0.1\rangle, \\
\widetilde{c}_{B} & =\langle(8,9,10) ; 0.8,0.1\rangle, \\
\widetilde{\alpha} & =\langle(70,71,72) ; 0.7,0.2\rangle, \\
\widetilde{\beta} & =\langle(0.10,0.11,0.12) ; 0.6,0.3\rangle .
\end{aligned}
$$

According to the analysis of Section 3, the optimum output quantities of the four patterns are shown in Table 2.

The fuzzy maximum profits and the fuzzy total profits of the four patterns are shown in Table 3. The optimum price of

\begin{tabular}{|c|c|}
\hline Enterprise & Pattern 1 \\
\hline$A$ & $\operatorname{TIF}_{F_{A 1}}^{*}=\langle(3215.56,4430.71,5675.85) ; 0.60,0.30\rangle$ \\
\hline$B$ & $\operatorname{TIF\Pi }_{F_{B 1}}^{*}=\langle(2230.52,3286.16,4381.80) ; 0.60,0.30\rangle$ \\
\hline Total & TIFח $_{1}^{*}=\langle(5446.09,7716.87,10057.65) ; 0.60,0.30\rangle$ \\
\hline Enterprise & Pattern 2 \\
\hline$A$ & $\operatorname{TIF}_{L_{A 2}}^{*}=\langle(3037.60,5014.55,7021.49) ; 0.60,0.30\rangle$ \\
\hline$B$ & $\operatorname{TIF}_{F_{B 2}}^{*}=\langle(731.90,1551.82,2411.74) ; 0.60,0.30\rangle$ \\
\hline Total & TIFח $_{2}^{*}=\langle(3769.50,6566.36,9433.22) ; 0.60,0.30\rangle$ \\
\hline Enterprise & Pattern 3 \\
\hline$A$ & $\operatorname{TIF}_{F_{A 3}}^{*}=\langle(1598.35,2624.20,3680.06) ; 0.60,0.30\rangle$ \\
\hline$B$ & $\operatorname{TIF}_{L_{B 3}}^{*}=\langle(2027.36,3725.68,5464.01) ; 0.60,0.30\rangle$ \\
\hline Total & TIFח $_{3}^{*}=\langle(3625.70,6349.89,9144.07) ; 0.60,0.30\rangle$ \\
\hline Enterprise & Pattern 4 \\
\hline$A$ & $\operatorname{TIF}_{L_{A 4}}^{*}=\langle(-2188.84,223.64,2666.12) ; 0.60,0.30\rangle$ \\
\hline$B$ & $\operatorname{TIF}_{L_{B 4}}^{*}=\langle(-2726.78,-632.27,1502.23) ; 0.60,0.30\rangle$ \\
\hline Total & $\mathrm{TIF \Pi}_{4}^{*}=\langle(-4915.62,-408.64,4168.35) ; 0.60,0.30\rangle$ \\
\hline
\end{tabular}
the four patterns is shown in Table 4.

By (2) and Table 2, we have that

$$
\begin{aligned}
& V_{\lambda}\left(\operatorname{TIF}_{F_{A 1}}^{*}\right)=4435.71 \times(0.49-0.13 \lambda), \\
& V_{\lambda}\left(\operatorname{TIF}_{L_{A 2}}^{*}\right)=5019.55 \times(0.49-0.13 \lambda),
\end{aligned}
$$

TABle 3: The fuzzy maximum profits and the fuzzy total profits of the four patterns.

$$
\begin{aligned}
& V_{\lambda}\left(\operatorname{TIF}_{F_{A 3}}^{*}\right)=2629.20 \times(0.49-0.13 \lambda), \\
& V_{\lambda}\left(\operatorname{TIF}_{L_{A 4}}^{*}\right)=228.64 \times(0.49-0.13 \lambda) .
\end{aligned}
$$


TABle 4: The optimum price of the four patterns.

\begin{tabular}{lc}
\hline Pattern 1 & Pattern 2 \\
\hline$\widetilde{p}_{1}^{*}=\langle(23.82,28.67,33.52) ; 0.60,0.30\rangle$ & $\tilde{p}_{2}^{*}=\langle(17.64,23.00,28.36) ; 0.60,0.30\rangle$ \\
\hline Pattern 3 & Pattern 4 \\
\hline$\widetilde{p}_{3}^{*}=\langle(18.45,23.75,29.05) ; 0.60,0.30\rangle$ & $\tilde{p}_{4}^{*}=\langle(0.73,7.50,14.27) ; 0.60,0.30\rangle$ \\
\hline
\end{tabular}

Then

$$
\begin{aligned}
V_{\lambda}\left(\operatorname{TIF}_{L_{A 2}}^{*}\right) & >V_{\lambda}\left(\operatorname{TIF}_{F_{A 1}}^{*}\right)>V_{\lambda}\left(\operatorname{TIF}_{F_{A 3}}^{*}\right) \\
& >V_{\lambda}\left(\operatorname{TIF}_{L_{A 4}}^{*}\right) .
\end{aligned}
$$

Therefore

$$
\operatorname{TIF}_{L_{A 2}}^{*}>\operatorname{TIF}_{F_{A 1}}^{*}>\operatorname{TIF}_{F_{A 3}}^{*}>\operatorname{TIF}_{L_{A 4}}^{*} .
$$

In the same way, the following results can be obtained:

$$
\begin{aligned}
\operatorname{TIF}_{L_{B 3}}^{*}>\mathrm{TIF}_{F_{B 1}}^{*}>\mathrm{TIF}_{F_{B 2}}^{*}>\operatorname{TIF}_{L_{B 4}}^{*}, \\
\mathrm{TIF}_{1}^{*}>\mathrm{TIF}_{2}^{*}>\mathrm{TIF}_{3}^{*}>\mathrm{TIF}_{4}^{*}, \\
\tilde{p}_{1}^{*}>\tilde{p}_{3}^{*}>\tilde{p}_{2}^{*}>\tilde{p}_{4}^{*} .
\end{aligned}
$$

Based on the analysis results stated above, some conclusions are be got as follows.

When both of enterprises $A$ and $B$ are followers, the equilibrium market price and the total fuzzy profit are maximal. When both of enterprises $A$ and $B$ are leaders, the equilibrium market price and the total fuzzy profit are minimal. When one is a leader, and the other is a follower; that is, enterprises $A$ and $B$ play Stackelberg game: the leader will obtain more profit than the follower. Therefore, the best decision is that both of enterprises $A$ and $B$ are followers.

5.2. Comparison Analysis. This section includes three aspects: firstly, the analysis of results about Section 5.1; secondly, the comparison of the results with Liang et al. (2008) [17]; and finally, the advantages of modeling fuzziness through TIFNs are discussed.

Firstly, based on the above conclusions in Section 5.1, we find the orders of the equilibrium market price and the total fuzzy profit in patterns 2 and 3 will exchange when some parameters are changing. For example, if $\widetilde{c}_{A}=$ $\langle(5,6,7) ; 0.9,0.1\rangle$ changes and the other parameters keep unchanging, the order of the fuzzy total profit is as follows:

$$
\operatorname{TIF\Pi }_{1}^{*}>\mathrm{TIF \Pi}_{3}^{*}>\mathrm{TIF \Pi}_{2}^{*}>\mathrm{TIF \Pi}_{4}^{*} .
$$

Because the market structures in patterns 2 and 3 are similar, by analyzing (22) to (31), the orders of the equilibrium market price and the total fuzzy profit in these two patterns are correlated with the position of $\left(\underline{c}_{A}+4 c_{A}+\bar{c}_{A}\right)$ and $\left(\underline{c}_{B}+\right.$ $\left.4 c_{B}+\bar{c}_{B}\right)$.

If

$$
\underline{c}_{A}+4 c_{A}+\bar{c}_{A}>\underline{c}_{B}+4 c_{B}+\bar{c}_{B}
$$

then

$$
\begin{aligned}
\operatorname{TIF}_{2}^{*} & <\operatorname{TIF}_{3}^{*}, \\
\tilde{p}_{2}^{*} & >\widetilde{p}_{3}^{*} .
\end{aligned}
$$

If

$$
\underline{c}_{A}+4 c_{A}+\bar{c}_{A}=\underline{c}_{B}+4 c_{B}+\bar{c}_{B}
$$

then

$$
\begin{aligned}
\operatorname{TIF\Pi }_{2}^{*} & =\operatorname{TIF\Pi }_{3}^{*}, \\
\widetilde{p}_{2}^{*} & =\widetilde{p}_{3}^{*} .
\end{aligned}
$$

Secondly, Liang et al. (2008) [17] designed a hypothetical optimum output quantity decision problem of duopoly market to explain the computational process. The cost functions and the parameters of market demand in [17] are triangular fuzzy numbers. Because TIFNs are an extension of triangular fuzzy numbers, those triangular fuzzy numbers can be rewritten to TIFNs. For example, the estimated fuzzy fixed costs for enterprise $A$ in [17] are $\widetilde{f}_{A}=(60000,65000,65000,68000)$, which can be rewritten to $\widetilde{f}_{A}=\langle(60000,65000,68000) ; 1,0\rangle$. Similarly, the estimated fuzzy fixed costs, fuzzy unit variable cost, and the parameters of market demand in [17] can be rewritten as follows:

$$
\begin{aligned}
\tilde{f}_{B} & =\langle(55000,60000,63000) ; 1,0\rangle, \\
\widetilde{c}_{A} & =\langle(150,200,230) ; 1,0\rangle, \\
\widetilde{c}_{B} & =\langle(200,250,280) ; 1,0\rangle, \\
\widetilde{\alpha} & =\langle(2100,2100,2100) ; 1,0\rangle, \\
\tilde{\beta} & =\langle(2,2,2) ; 1,0\rangle .
\end{aligned}
$$

Similar to the analysis in Section 5.1, the orders of fuzzy profits and total fuzzy profit for enterprises $A$ and $B$ are as below:

$$
\begin{gathered}
\operatorname{TIF}_{L_{A 2}}^{*}>\operatorname{TIF}_{F_{A 1}}^{*}>\operatorname{TIF}_{F_{A 3}}^{*}>\operatorname{TIF}_{L_{A 4}}^{*}, \\
\operatorname{TIF}_{L_{B 3}}^{*}>\operatorname{TIF}_{F_{B 1}}^{*}>\operatorname{TIF}_{F_{B 2}}^{*}>\operatorname{TIF}_{L_{B 4}}^{*}, \\
\operatorname{TIF}_{1}^{*}>\operatorname{TIF}_{2}^{*}>\operatorname{TIF\Pi }_{3}^{*}>\operatorname{TIF\Pi }_{4}^{*} .
\end{gathered}
$$

From the analysis results, the conclusions are in accordance with [17]. That means the approach using TIFNs in this paper is feasible to solve the optimum output quantity problem in practical application and is an extension method of the approach used in [17].

Thirdly, the advantages of the proposed model in this paper are analyzed. The effect of the degree of membership, the degree of nonmembership, and $\lambda$ on the fuzzy maximum profit of enterprise will be discussed based on the above example at first. And we suppose that the triangular intuitionistic fuzzy variables to be analyzed are the same as the above 
example used in [17], but the degree of membership and the degree of nonmembership are changing.

Let us take enterprise $A$ in pattern 1 as an example. According to (9) and (17), the $V_{\lambda}\left(\operatorname{TIF}_{F_{A 1}}^{*}\right)$ of $\operatorname{TIF}_{F_{A 1}}^{*}$ can be found as follows:

$$
\begin{aligned}
V_{\lambda}\left(\operatorname{TIF}_{F_{A 1}}^{*}\right)= & 147306 \\
& \times\left[\lambda \omega_{\widetilde{A}}^{2}+(1-\lambda)\left(1-u_{\widetilde{A}}\right)^{2}\right] .
\end{aligned}
$$

Taking the partial derivative of $V_{\lambda}\left(\operatorname{TIF}_{F_{A 1}}^{*}\right)$ with respect to $\omega_{\widetilde{A}}, u_{\widetilde{A}}$, and $\lambda$, respectively,

$$
\begin{aligned}
& \frac{\partial V_{\lambda}\left(\operatorname{TIF}_{F_{A 1}}^{*}\right)}{\partial \omega_{\widetilde{A}}}=294612 \times \omega_{\widetilde{A}} \times \lambda \geq 0, \\
& \frac{\partial V_{\lambda}\left(\operatorname{TIF}_{F_{A 1}}^{*}\right)}{\partial u_{\widetilde{A}}}=294612 \times(1-\lambda)\left(u_{\widetilde{A}}-1\right) \leq 0, \\
& \frac{\partial V_{\lambda}\left(\operatorname{TIF} \Pi_{F_{A 1}}^{*}\right)}{\partial \lambda}=147306 \times\left[\omega_{\widetilde{A}}^{2}-\left(1-u_{\widetilde{A}}\right)^{2}\right] \leq 0,
\end{aligned}
$$

which means that the fuzzy maximum profit of enterprise $A$ in pattern 1 increases in $\omega_{\widetilde{A}}$ but decreases in $u_{\widetilde{A}}$ and $\lambda$.

Similarly, we can also analyze the effect of these three fuzzy variables on the fuzzy maximum profit of enterprise $A$ for the other three patterns or enterprise $B$ for four patterns in the same way. Therefore, we can find that the degree of membership or nonmembership of TIFNs and the preference information of decision makers $(\lambda)$ will affect the results of the fuzzy maximum profit.

Based on the above analysis, the approach using trapezoidal fuzzy numbers proposed in [17] and the approach using TIFNs in this paper will be compared, and some differences and advantages of the proposed fuzzy approach in this paper will be found as follows.

Firstly, the difference between this two approaches is the way authors treat the fuzziness of the data. In [17], the author used trapezoidal fuzzy numbers to handle the fuzziness of the decision variables; however, TIFNs are used in this paper to describe the market uncertainty. Intuitionistic fuzzy numbers are an extension of fuzzy numbers, which can depict comprehensively the fuzzy essence of uncertainty information by the degree of membership and nonmembership. Therefore, the approach in this paper is better than in [17]. Secondly, the fixed cost and unit variable cost are described by fuzzy numbers without considering the parameters of inverse demand function in [17]. However, the inverse demand functions of market and the cost functions are both described by TIFNs in this paper, which can be better to describe uncertain market and improve the realism of the model. Thirdly, according to Section 5.2, we find that the degree of membership, the degree of nonmembership of TIFNs, and the preference information of decision makers $(\lambda)$ have effect on the results of the fuzzy maximum profit. Therefore, introducing TIFNs and $\lambda$ to construct fuzzy model in this paper can depict fuzzy preference information of decision makers and be convenient for managers or decision makers to make decision for their enterprise.

\section{Conclusions}

Due to the influences of some factors, such as paucity of data and ambiguous environment, it is not easy to obtain the exact economic assessment of data in the real world situation. The intuitionistic fuzzy numbers are just the suitable tool to express these ill-known quantities. Thus, in this paper, we apply TIFNs to solve the fuzziness aspect of demand and cost uncertainty for two enterprises. For simplicity, we assume that the inverse demand and cost functions of enterprises are TIFNs behaving in a linear form. The equilibrium quantity of each enterprise in a competitive market can be found. Furthermore, we conduct a sensitivity analysis to discuss the impacts of fuzzy parameter on optimum output quantity and market demand for the four competitive behaviors. Finally, a numerical example is given and the comparison between two approaches is analyzed, concluding three advantages of the proposed model at last.

In order to obtain optimum output quantities of enterprises, it is needed to defuzzify the fuzzy profit function into a crisp value. In this paper, we only use the value-index that proposed in [21] to defuzzify the fuzzy profit function. Some results proposed in this paper showed that the degree of membership or nonmembership of TIFNs and the preference information of decision makers cannot adequately reflect some characteristics of TIFNs, such as the results of output quantity or the sequence of the fuzzy profit in four patterns for one enterprise. Therefore, how to find a good defuzzification method of a fuzzy function is also an important issue in the future.

\section{Conflict of Interests}

The authors declare that there is no conflict of interests regarding the publication of this paper.

\section{Acknowledgments}

The work on this paper is supported by National Natural Science Foundation of China (no. 71401003), Ministry of Education in China Project of Humanities and Social Sciences (nos. 14YJC630114 and 11YJC630203), Bases for Scientific Research-Science and Technology Innovation PlatformModern Business Features (PXM2015014213000063), and Scientific Research Capacity Enhancing Project of Postgraduates of Beijing Technology and Business University in 2015.

\section{References}

[1] J. C. Cox and M. Walker, "Learning to play cournot duopoly strategies," Journal of Economic Behavior and Organization, vol. 36, no. 2, pp. 141-161, 1998.

[2] H. von Stackelberg, Marktform und gleichgewicht, Julius Springer, 1934.

[3] J.-F. Dang, I.-H. Hong, and J.-M. Lin, "The cournot production game with multiple firms under an ambiguous decision environment," Information Sciences, vol. 266, pp. 186-198, 2014. 
[4] A. Van den Berg, I. Bos, P. J.-J. Herings, and H. Peters, "Dynamic cournot duopoly with intertemporal capacity constraints," International Journal of Industrial Organization, vol. 30, no. 2, pp. 174-192, 2012.

[5] J. Barr and F. Saraceno, "Cournot competition, organization and learning," Journal of Economic Dynamics and Control, vol. 29, no. 1-2, pp. 277-295, 2005.

[6] P. Guo, "One-shot decision approach and its application to duopoly market," International Journal of Information and Decision Sciences, vol. 2, no. 3, pp. 213-232, 2010.

[7] P. Guo, R. Yan, and J. Wang, "Duopoly market analysis within one-shot decision framework with asymmetric possibilistic information," International Journal of Computational Intelligence Systems, vol. 3, no. 6, pp. 786-796, 2010.

[8] L. Colombo and P. Labrecciosa, "On the convergence to the Cournot equilibrium in a productive asset oligopoly," Journal of Mathematical Economics, vol. 49, no. 6, pp. 441-445, 2013.

[9] Y. Fang and B. Shou, "Managing supply uncertainty under supply chain Cournot competition," European Journal of Operational Research, vol. 243, no. 1, pp. 156-176, 2015.

[10] M.-C. Hu, Y.-H. Chen, and L.-C. Huang, "A sustainable vegetable supply chain using plant factories in Taiwanese markets: a Nash-Cournot model," International Journal of Production Economics, vol. 152, pp. 49-56, 2014.

[11] V. DeMiguel and H. Xu, "A stochastic multiple-leader Stackelberg model: analysis, computation, and application," Operations Research, vol. 57, no. 5, pp. 1220-1235, 2009.

[12] D. De Wolf and Y. Smeers, "A stochastic version of a StackelbergNash-Cournot equilibrium model," Management Science, vol. 43, no. 2, pp. 190-197, 1997.

[13] T. Nakamura, "One-leader and multiple-follower Stackelberg games with private information," Economics Letters, vol. 127, pp. 27-30, 2015.

[14] S. Kim, "Multi-leader multi-follower stackelberg model for cognitive radio spectrum sharing scheme," Computer Networks, vol. 56, no. 17, pp. 3682-3692, 2012.

[15] L. J. Alvarez-Vázquez, N. García-Chan, A. Martínez, and M. E. Vázquez-Méndez, "Stackelberg strategies for wastewater management," Journal of Computational and Applied Mathematics, vol. 280, pp. 217-230, 2015.

[16] S.-L. Yang and Y.-W. Zhou, “Two-echelon supply chain models: considering duopolistic retailers' different competitive behaviors," International Journal of Production Economics, vol. 103, no. 1, pp. 104-116, 2006.

[17] G.-S. Liang, L.-Y. Lin, and C.-F. Liu, "The optimum output quantity of a duopoly market under a fuzzy decision environment," Computers and Mathematics with Applications, vol. 56, no. 5, pp. 1176-1187, 2008.

[18] M.-H. Shu, C.-H. Cheng, and J.-R. Chang, "Using intuitionistic fuzzy sets for fault-tree analysis on printed circuit board assembly," Microelectronics Reliability, vol. 46, no. 12, pp. 21392148, 2006.

[19] D.-F. Li, "A ratio ranking method of triangular intuitionistic fuzzy numbers and its application to MADM problems," Computers and Mathematics with Applications, vol. 60, no. 6, pp. 1557-1570, 2010.

[20] D. F. Li, "A note on 'using intuitionistic fuzzy sets for fault-tree analysis on printed circuit board assembly"', Microelectronics Reliability, vol. 48, no. 10, 1741 pages, 2008.

[21] D. F. Li, J. X. Nan, and M. J. Zhang, "A ranking method of triangular intuitionistic fuzzy numbers and application to decision making," International Journal of Computational Intelligence Systems, vol. 3, no. 5, pp. 522-530, 2010. 


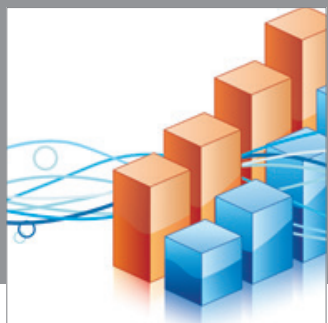

Advances in

Operations Research

mansans

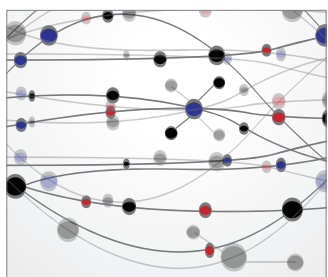

The Scientific World Journal
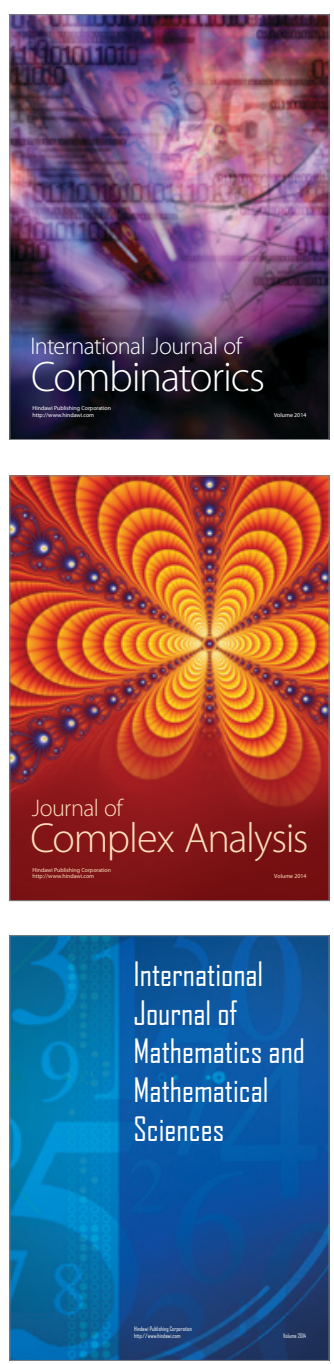
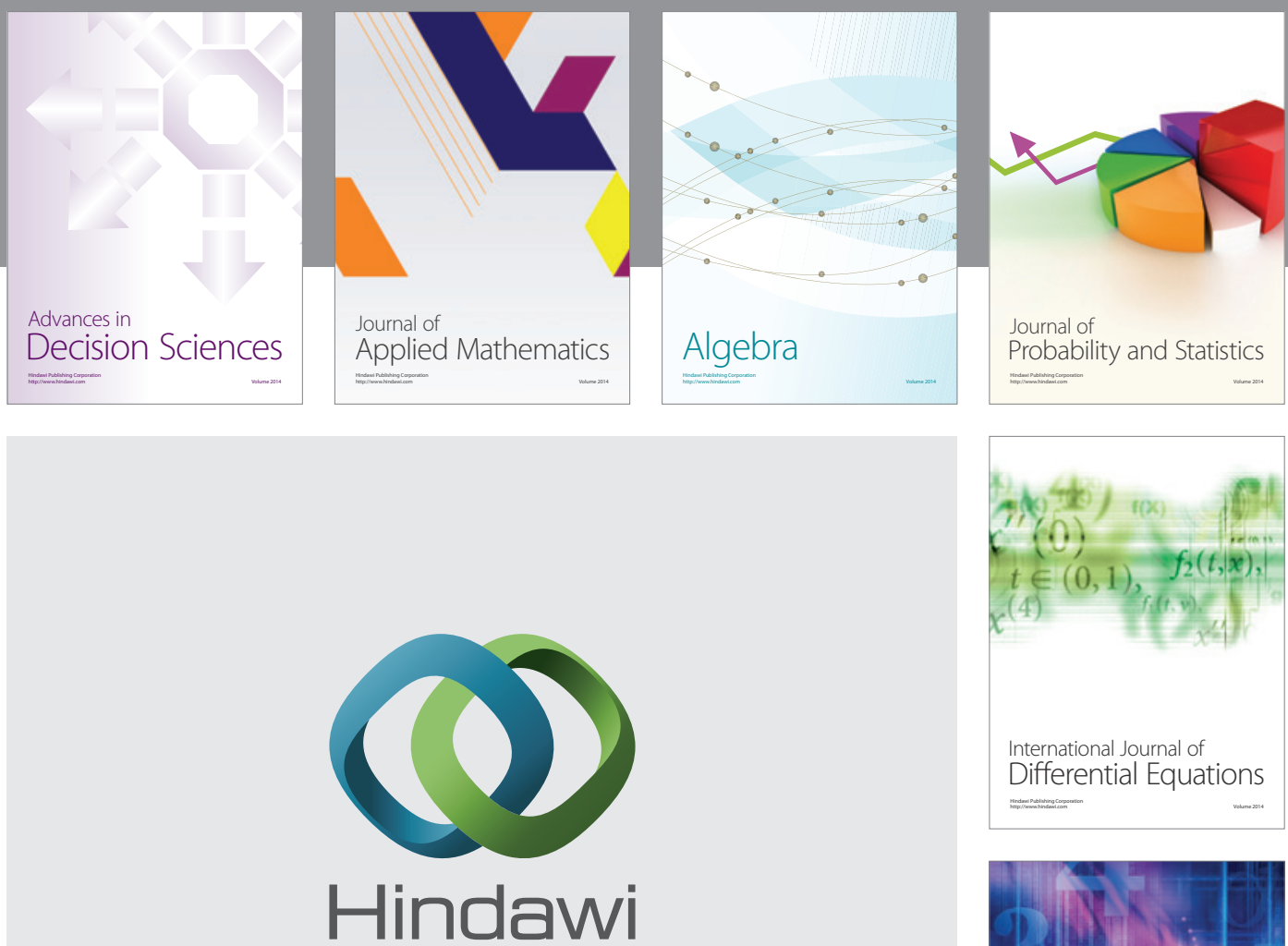

Submit your manuscripts at http://www.hindawi.com
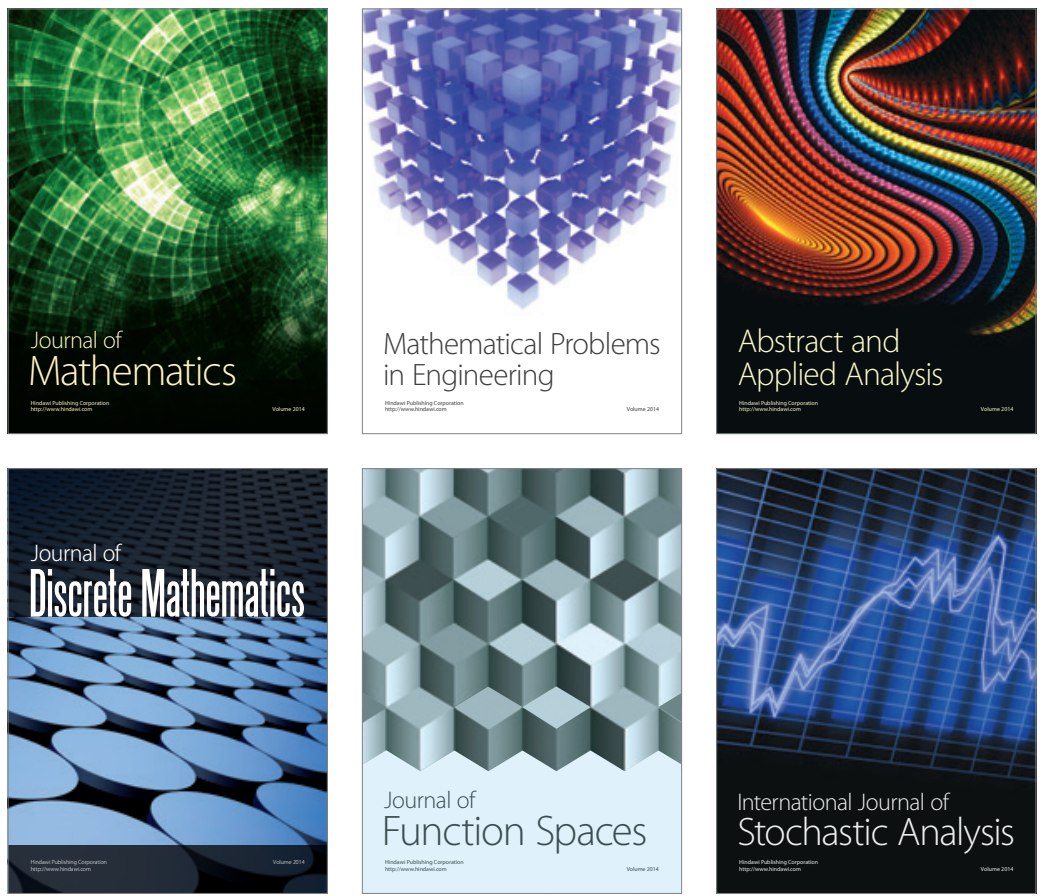

Journal of

Function Spaces

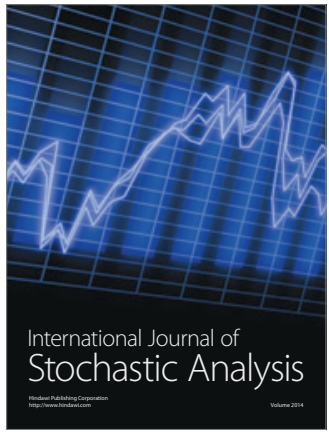

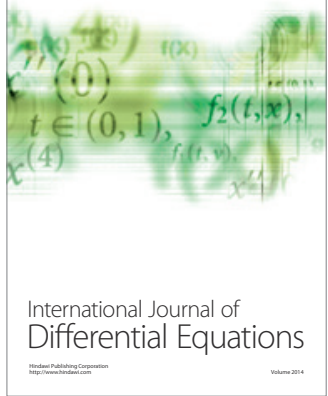
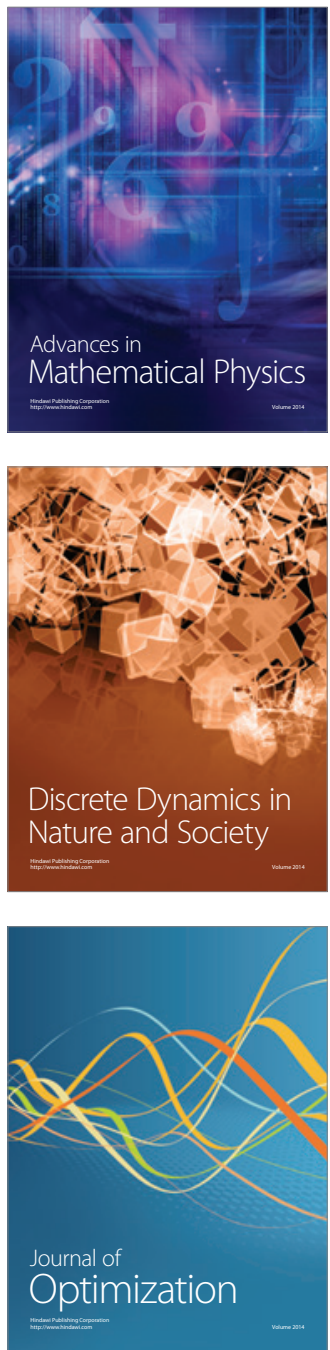